\title{
Assessing uncertainty in past ice and climate evolution: overview, stepping-stones, and challenges
}

\author{
Lev Tarasov ${ }^{1}$ and Michael Goldstein ${ }^{2}$ \\ ${ }^{1}$ Department of Physics and Physical Oceanography, Memorial University of Newfoundland and Labrador, St. John's, \\ Canada, A1B 3X7 \\ ${ }^{2}$ Durham University, Durham, England
}

Correspondence: Lev Tarasov (lev@mun.ca)

\begin{abstract}
.
In the geosciences, complex computational models have become a common tool for making statements about past earth system evolution. However, the relationship between model output and the actual earth system (or component thereof) is generally poorly specified and even more poorly assessed. This is especially challenging for the paleo sciences for which data constraints are sparse and have large uncertainties. Bayesian inference offers a self-consistent and rigorous framework for assessing this relationship as well as a coherent approach to combining data constraints with computational modelling. Though "Bayesian" is becoming more common in paleoclimate and paleo ice sheet publications, our impression is that most scientists in these fields have little understanding of what this actually means nor are they able to evaluate the quality of such inference. This is especially unfortunate given the correspondence between Bayesian inference and the classical concept of the scientific method.

Herein, we examine the relationship between a complex model and a system of interest, or in equivalent words (from a statistical perspective), how uncertainties describing this relationship can be assessed and accounted for in a principled and coherent manner. By way of a simple example, we show how inference can be severely broken if uncertainties are erroneously assessed. We explain and decompose Bayes Rule (more commonly known as Bayes Theorem), examine key components of Bayesian inference, offer some more robust and easier to attain stepping stones, and provide suggestions on implementation and how the community can move forward. This overview is intended for all interested in making and/or evaluating inferences about the past evolution of the Earth system (or any of its components), with a nominal focus on past ice sheet and climate evolution during the Quaternary.
\end{abstract}


https://doi.org/10.5194/cp-2021-145

Preprint. Discussion started: 17 November 2021

(c) Author(s) 2021. CC BY 4.0 License.

(c) (i)

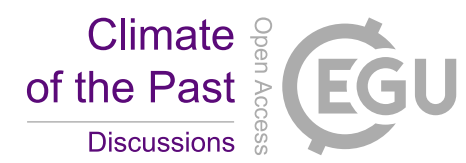

\section{Introduction}

"No one trusts a model except the [wo]man who wrote it; everyone trusts an observation except the [wo]man who made it", Harlow Shapely.

For those studying the past evolution of the earth/climate system, a key activity is to make inferences about it evolution and understand the processes underlying this evolution. However beyond inferences closed tied to well-constrained proxies and/or to core physics of the ice/climate/earth system, asking "what do we really know" and especially "how certain are we" is a challenge to answer.

Given the relatively sparse (in both time and space) data from the past along with uncertainties in their interpretation and dating, large-scale inferences nominally based solely on paleo data often arguably rely heavily on storytelling with varying combinations of proxy models relating a datum to a physical characteristic of interest, age calibration models, and basic physical reasoning for self-consistency. Evolving computational models of various components of the Earth and climate system permit data-rich stories that incorporate the embodied physical equations (or more accurately approximations thereof). But when used on their own, they have limited retrodictive confidence. They therefore also arguably fall within the storytelling and self-consistency rubric. It is also our own experience that modellers who know their models well are often the most skeptical about their model results, contrary to Harlow Shapely's quip quoted above.

The exponential growth of accessible computer power over the last few decades has permitted the ongoing development of a synthesis of the above two approaches: inference based on rigorously combining computational modelling with paleo observations. This potentially offers detailed pictures of ice sheet and climate system evolution that can go confidently well beyond storytelling but, as detailed below, only when all uncertainties are rigorously addressed and assessed. Such assessment is to date not prevalent within the community (though relevant communities are making calls for such assessment, e.g., Whitehouse and Tarasov, 2014; Stokes et al., 2015) and is the central theme of this overview.

Story-telling, or in more usual terminology, hypothesis creation/elaboration, is a central part of science. This is a characteristic common to most (all?) disciplines that endeavour to explain the world around us, including history and theology. What distinguishes the scientific enterprise? Though the philosophy of science offers nuanced alternatives, we posit most scientists would assign a central role to the rigorous testing of falsifiable hypotheses (and continuous testing of theories) as one distinguishing feature. However, without rigorous measures to quantify the correspondence between model-based realizations of hypotheses and the "real world", such testing becomes vague and ill-defined. Such correspondence quantification, aka uncertainty assessment, enables meaningful statements about the world around us instead of statements that only describe the behaviour of our models..

Given the above, rigorous quantification of uncertainty is, we submit, another distinguishing feature of the natural sciences.

50 It is also a feature that deserves more attention in relevant "culture wars" and in dealing with anti-science memes. However, too often, uncertainties are either ignored, or only partially assessed. Furthermore, as we'll elucidate below, the assumptions underlying commonly utilized statistics are generally broken for the given context. Another tendency is to provide various 
provisos such as "subject to unaccounted uncertainties in the climate forcing" that somehow the average reader is supposed to be better able to assess/interpret the impact thereof than the modeller who should be most intimately familiar with their model's foibles.

As we will show below, incomplete uncertainty assessment will result in inferences that are incorrect with, for example, "reality" well outside of the conventional two sigma, uncertainty bounds. Rigorous uncertainty assessment is a challenging task for many modelling contexts, especially all dealing with complex environmental systems. It has attracted significant attention from philosophers of science (e.g., Frigg et al., 2015b). It is also a task that can't be ignored as any natural scientist needs to be able to assess the extent and rigour of uncertainty assessment as they review and assimilate literature. However most in the natural sciences are not provided with the appropriate conceptual tools for such assessment during their training.

A related issue in the context of data model comparison is the tendency to focus attention on simulations that best fit observational/paleo data with little or no attention to what may be gleaned from other simulations within (and outside of) inferential bounds. By more clearly and credibly defining the uncertainty bounds of one's inferences, one can better delineate what is not known and where future efforts might offer the most scientific return. Accepting this premise would require much more attention on, for example, the max/min bounds of ice sheet evolution during the last deglaciation. Yet major international endeavours such as the paleo model intercomparison project (PMIP) have, to date, only based their intercomparisons on using "best-fitting" ice sheet chronologies as their boundary conditions, ignoring the uncertainties in these chronologies (some of which have no assessed uncertainties).

This overview is intended for all who are concerned with either inferring past ice sheet and/or climate system evolution or using the results of such inferences (be they modellers, data gatherers, or mongrels). Our goal is that after a careful read, you will at least be able to more critically evaluate uncertainty assessment for such contexts. This includes gaining some awareness of common statistical errors underlying virtually all paleo modelling studies to date that purport to infer past system evolution. Much of the discussion should also be relevant to those interested in inferring other aspects of the paleo environment for contexts that can benefit from rigorous integration of modelling and paleo data. The presentation is largely conceptual given the breadth of the intended audience. However, clearly delineated sections offer more specific and technical guidance for those interested in actual implementation and further development of relevant methodologies.

Below, we lay out a broad framework for uncertainty quantification (Sect. 2), consider a few stepping-stones (Sect. 3), provide guidance on implementation (Sect. 4), and suggest some key concrete steps we believe are necessary for the community to move forward (Sect. 5). The framework necessarily follows a Bayesian approach. We know of no other approach to uncertainty quantification that can rigorously determine a meaningful assessment of probability of, for instance, mean sea level having a specific value at last glacial maximum. Given the still prevalent lack of understanding of Bayesian inference among much of the paleo science community, the presentation begins with a high-level introduction to Bayesian inference for the paleo context. 


\section{2 the Bayesian framework}

This section provides the conceptual framework for Bayesian inference by considering each term in Bayes theorem in turn along with an examination of how distinct sources of uncertainty can be meaningfully assessed. It also provides a simple example of how inference can be broken when uncertainties are not appropriately addressed. The correspondence between Bayesian inference and the scientific method is underlined and should aid the reader in the understanding of Bayes theorem. None of these issues can be well addressed without consideration of what "probability" means, to which we first turn.

\section{1 "What is Probability?"}

In addressing uncertainty, the issue of what is probability is unavoidable. Is it purely an individual's judgment, subject to the rules of probability theory, about how likely some inference or event is? This is crudely the personalistic interpretation of probability. Or does it reflect something more objective? If so, what does "objective" mean? To what extent are probabilities independent properties of the world? When you make a probabilistic statement concerning your own research, do you believe your statement (and not just in the sense "this is what follows if I apply this statistical tool")? What does your probabilistic statement mean to you? How does it actually apply to the world around us? Or perhaps to be more concrete, how much of one week's salary would you bet that your assertion is true with the odds that follow from your probabilistic statement? ${ }^{1}$

No matter what interpretation of probability one chooses, the assignment of probabilities require judgements. To be testable and potentially falsifiable, these judgements must be made and treated in a rigorous and self-consistent way. A natural way to achieve this is to require that probabilities follow the rules of probability theory (e.g., Kolmogorov, 1933). Specifically:

1. The probability of an event is a non-negative real number.

2. An impossible event has 0 probability ${ }^{2}$.

3. A certain event has probability of 1 .

4. Probability of events $A$ or $B$ is equal to the sum of their individual probabilities $(P(A$ or $B)=P(A)+P(B)$ when $A$ and $B$ are disjoint, i.e. one can happen only when the other can't happen ${ }^{3}$.

Adherence to the rules of probability ensures that once required judgements are transparently made, all ensuing probabilistic calculations are acceptable to all competent practitioners given the premises (even if the subsequent interpretations may vary). A key consequence of the rules of probability is Bayes rule.

\footnotetext{
${ }^{1}$ This is an informal version of a precise statement based on the use of coherent scoring rules. Note: to partly address the challenge of maintaining both statistical rigour and accessibility for readers with limited statistical understanding, we rely on footnotes for those concerned about the former.

${ }^{2}$ This formally follows from the other three rules but is included for the sake of a non-statistical audience

${ }^{3}$ For the mathematically inclined, it should also be noted that countable additivity is usually added.
} 
https://doi.org/10.5194/cp-2021-145

Preprint. Discussion started: 17 November 2021

(c) Author(s) 2021. CC BY 4.0 License.

(c) (1)

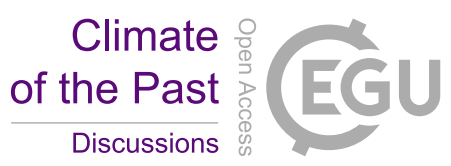

\subsection{Bayesian inference for the paleo community: the scientific method expressed in probability theory}

Bayesian inference is concerned with deriving rigorous inferential probabilities given current knowledge and new data. This contrasts with traditional frequentist statistics which does not directly determine inferential probabilities and necessarily assumes repeated experiments. Bayesian inference is encapsulated in Bayes rule itself:

Posterior probability $=\frac{\text { Likelihood } \times \text { Prior probability }}{\text { normalization constant }}$

The unpacking of what each of the terms mean and how they are determined will proceed below with dedicated subsections for each term on the right-hand side. As a start, understanding of some basic notation is required. $P(A=a \mid B=b)$ denotes the conditional probability of the variable A having some specific value "a" if the statement that the variable B has some value "b" were true. This is usually abbreviated to $P(A \mid B)$ when considering the probabilistic relationships independent of any specific value $\mathrm{a}$ or $\mathrm{b}$ (from now on a lower case letter will refer to a specific value of the corresponding upper case variable). $P(A, B)$ is the joint probability of $A$ and $B, e . g$., the probability that $A=a$ AND $B=b$. To be consistent under various sets of natural axioms, the conditional probability $P(A \mid B)$ can be defined as the quotient of the joint probability of $A$ and $B, P(A, B)$, and the probability of $B, P(B)$ :

$P(A \mid B)=\frac{P(A, B)}{P(B)}$

To concretize these equations, consider $A$ having two values: rain $(A=$ rain) or no rain $(A=$ no rain) tomorrow afternoon. Let $B$ also have two values: cloudy $(B=$ cloudy) or clear sky $(B=$ clear sky) conditions tomorrow morning. Then the conditional probability that tomorrow afternoon will have rain if tomorrow morning is cloudy, $P$ ( $A=\operatorname{rain} \mid B=$ cloudy), is equal to the joint probability of rain tomorrow afternoon and cloudy skies tomorrow morning, $\mathrm{P}(A=$ rain, $B=$ cloudy $)$, divided by the probability of cloudy skies tomorrow morning ( $P(B=$ cloudy). The crucial difference between joint and conditional probability is that the former has no input condition, while the later has the given condition (i.e. that the statement $B=$ cloudy is true in our example).

Multiplying both sides of equation 2 by $P(B)$ gives the multiplication rule for probabilities:

The above may also provide a more understandable implicit definition for conditional probability, i.e. $P(A \mid B)$ being that quantity which when multiplied by $P(B)$ provides the joint probability $P(A, B)$. An important self-consistency feature that we will use below is that the multiplication rule holds even if an additional conditional variable is added to both sides of the above equation, as follows by repeated use of the definition of conditional probability (equation 1), sic:

$$
\begin{aligned}
P(A, B \mid C) & =\frac{P(A, B, C)}{P(C)} \\
& =\frac{P(A, B, C)}{P(B, C)} \frac{P(B, C)}{P(C)} \\
& =P(A \mid B, C) P(B \mid C)
\end{aligned}
$$


To place Bayes rule in a more useful formulaic representation, consider a set of variables $\left(C_{M}\right.$, which we'll refer to as the parameter vector) that collectively defines the current configuration of a model. For a computational model, $C_{M}$ includes model parameters (such as basal drag coefficients for sliding ice in an ice sheet model, coefficients controlling cloud formation in climate models,...). $C_{M}$ can also define the selection of external forcings or inputs (e.g., to define parametrized aspects of the climate history that drives a glaciological model not coupled to a dynamical climate model). We are interested in finding a probability distribution for which value of $C_{M}$ is most appropriate ${ }^{4}$. Prior to testing the model against new data, one's initial (or previously derived) judgement for the probability distribution of $C_{M}$ is denoted by $P\left(C_{M}\right)$. The revised (posterior) probability distribution for $C_{M}$ given comparison of new data $(D)$ against model results is given by Bayes rule (or conceptually in above eq. 1):

$P\left(C_{M} \mid D\right)=\frac{P\left(D \mid C_{M}\right) P\left(C_{M}\right)}{P(D)}$

Bayes rule follows from the given definition for conditional probability (eq. 2) or equivalently from the axiomatic multiplication rule):

$$
\begin{aligned}
P\left(C_{M} \mid D\right) & =\frac{P\left(C_{M}, D\right)}{P(D)} \\
& =\frac{P\left(D \mid C_{M}\right) P\left(C_{M}\right)}{P(D)}
\end{aligned}
$$

From Bayes rule, the posterior probability for a single parameter vector $C_{M}$ given data $D$ is proportional to the product of the likelihood $\left(P\left(D \mid C_{M}\right)\right)$ and prior probability $\left(P\left(C_{M}\right)\right)$. It should be noted that there is also implicit dependence on all the assumptions and judgements underlying one's use of Bayes rule ${ }^{5}$.

To make the above concrete, consider our previous rain and cloud example. Let $C_{M}$ represent our "model" for whether there will be rain in the afternoon and that it can have only two values (Rain or noRain). Let $D$ be the datum of whether the sky is clear or cloudy when we look out the window after waking up. Suppose based on climatology, and the fact that we had no rain yesterday, our prior, i.e. before looking out the window, is $P\left(C_{M}=\right.$ Rain $)=0.3$ (and therefore $P\left(C_{M}=\right.$ NoRain $)=0.7$ ). Further suppose our likelihood has the following values:

$$
P\left(D=\text { clear } \mid C_{M}=\text { Rain }\right)=0.1,
$$

$$
P\left(D=\text { clear } \mid C_{M}=\text { NoRain }\right)=0.8,
$$

\footnotetext{
${ }^{4}$ For the purposes of this and the next few subsections, we make the simplifying assumption that there is a value of the parameter choices (which we don't know) that is sufficient: once the model is run with that choice, no more information about the system would be gained by running the model with a different choice of parameters. We will take a more nuanced view in Sect. 3.1

${ }^{5}$ In the above and throughout, if variables are continuous, probabilities would be replaced by corresponding probability density functions.
} 
with the remaining two values given by the difference from one (e.g., $P\left(D=\right.$ cloudy $\mid C_{M}=$ Rain $\left.)=1-0.1=0.9\right)$. After we look out the window and see that the sky is clear, our posterior for rain this afternoon is :

$$
\begin{aligned}
P\left(C_{M}=\text { Rain } \mid D=\text { clear }\right) & =\frac{P\left(D=\text { clear } \mid C_{M}=\text { Rain }\right) \times P\left(C_{M}=\text { Rain }\right)}{P(D)} \\
& =\frac{0.1 \times 0.3}{0.1 \times 0.3+0.8 \times 0.7}=0.05
\end{aligned}
$$

The value used for $P(D)$ follows $^{6}$ from requiring that $P\left(C_{M}=\right.$ Rain $\mid D=$ clear $)+P\left(C_{M}=\right.$ NoRain $\mid D=$ clear $)=1$.

To facilitate a broader interpretation of Bayes rule, consider the idealized form of the scientific method as taught in primary school science or described in Wikipedia. In detail, start with a hypothesis based on current understanding, carry out an experiment, and thereby test the hypothesis and revise our understanding. Bayes rule translates to this description of the scientific method as follows. Start with a prior understanding and considered judgement of the probability distribution for a given model configuration $\left(P\left(C_{M}\right)\right)$. Get new data $D$ (from a data archive, laboratory experiment, or fieldwork). Compare this data to model predictions via the likelihood function $\left(P\left(D \mid C_{M}\right)\right)$, which is the conditional probability of the data given the parameter vector (and therefore given the model as the parameter vector has no meaning without an associated model). Hence revise one's understanding (update the probability distribution for model parameters and/or competing models) of the phenomena under consideration as expressed in the posterior, $P\left(C_{M} \mid D\right)$, i.e. the conditional probability of the parameter vector (and model) given the new data. The likelihood comparison embodies the experimental test of the model.

"Model" is any approximate representation of some aspect of the world around us (past/present/future), and thereby encompasses not only computer-based models, but also conceptual models including scientific hypotheses and theories. For nonmodellers, the evolving sedimentation rate of a marine or lake core and associated chronology is perhaps a conceptually closer example of a model. Where relevant, we will adopt the convention of specifically denoting an implemented computational model by "simulator" (to differentiate from the set of physical equations that the simulator is approximating).

Given this correspondence to the scientific method, Bayes rule provides a core probabilistic underpinning for the natural sciences. It should therefore arguably be both understood and used by all scientists whose work involves comparison between data and models (in the previously presented most general sense of "model"). To pursue this further, each term in Bayes rule is detailed below. Those interested in a much more thorough introduction are referred to one or both of the following texts: Sivia and Skilling (2006); Gelman et al. (2013). Rougier (2007) provides a complementary presentation to much of this section for a general climate modelling context. Kennedy and O’Hagan (2001) is a good starting point for a standard Bayesian approach to uncertainty in computer models.

\subsection{Defining the prior}

The prior $\left(P\left(C_{M}\right)\right)$ embodies one's initial understanding of the system in question. Initially, before the first comparison between simulator prediction and data, the prior will be formulated on the basis of explicit user/expert judgement, likely in combination with results from other studies. The initial prior for a single model parameter, for instance, may be judged to be a trapezoidal

\footnotetext{
${ }^{6}$ It directly follows from the law of total probability.
} 


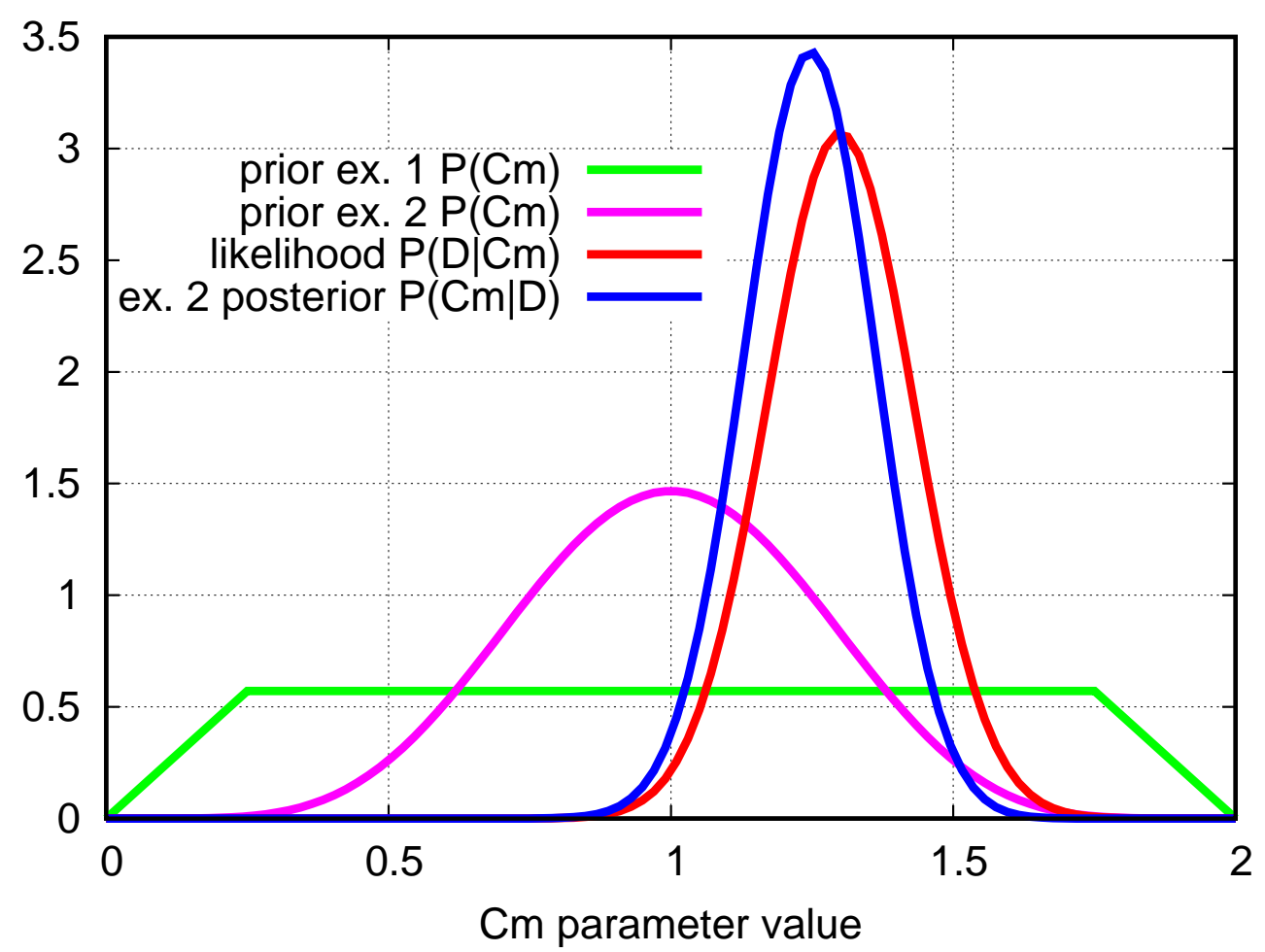

Figure 1. Example prior and posterior probability distributions for a parameter vector consisting of a single parameter $\left(C_{M}\right)$ with maximum physically plausible range of 0 to 2 . The likelihood (red) is also the posterior for prior example 1 . Prior example 2 (purple) subject to the red likelihood results in the blue posterior.

distribution over a parameter range encompassing physically plausible values, an example of which is shown by the example 1 prior in green in Fig. 1. For the example of a marine core, the prior sedimentation rate would require expert judgement taking into account inferred rates from proximal cores, possible range of sedimentation variations given plausible ranges on biological productivity,...

This judgement aspect has often been a target by critics of Bayesian approaches, with a usual focus on the specification of the prior. This focus has no clear justification as judgements are required for all aspects of the inferential process and not just the initial specification of the prior. But this holds true for any statistical inference including those by frequentist approaches. For instance, many scientists will rely on standard frequentist statistics such as $\chi^{2}$, confidence intervals from standard deviations, p-value tests, ... These often presume an underlying Gaussian structure to the uncertainties which all too frequently is not explicitly stated let alone tested. Judgements in the choice of constraint data, simulator, and which simulator parameters will be calibrated are required under any inferential approach. More fundamentally, traditional frequentist approaches do not offer a route to a rigorous probabilistic inference about past ice or climate (in part because the frequency of occurrence of a single 
possible non-repeated event has no meaning within the frequentist framework). Furthermore, the frequentist approach does not allow a direct inference about a model given the data. It instead makes inferences about the data, assuming that a model is true.

Bayesian approaches tend to force the user to make all such assumptions explicit. Furthermore, for a sufficiently wide prior, the impact of the prior on the posterior diminishes as the specificity of the likelihood increases (i.e. as the constraint value of the data increases). For the example in Fig. 1, this would translate into reduced posterior dependence on the choice between the two indicated priors with increased specificity (narrowness in the plot) of the likelihood.

Prior specification should therefore err on a clearly ample range over which the quantity of interest has non-negligible probability. A prior that is set narrow without good reason may exclude a high probability region of the posterior resulting from a prior that was more carefully specified. The trade-off is that excessively wide priors increase the computational cost of the inference procedure. Thus the effort put into prior specification should be proportional to the computational expense of the simulator, and inversely proportional to the amount and quality of constraint data.

A key self-consistent feature of Bayes rule is that it can be applied sequentially, with the posterior from an initial application of the rule becoming the prior for a subsequent application with new data (and therefore a new likelihood, as shown in eq. 6). Thus, after initial formulation of the prior, its subsequent value for the same simulator for use with new data is rigorously set by the Bayesian inferential process. For our sedimentation example, an existing posterior inference for sedimentation rates could become the prior for a revised posterior from likelihood application of new $\mathrm{C} 14$ dates.

The judgement aspect of prior (and likelihood) specification does not imply all choices of prior specification are equivalent. Prior specification must obey the rules of probability. It must also be transparent, respect physical bounds, and be defensible to the relevant community of experts (all normative criteria for "good" science). "Defensible" does not mean that everyone will make the same assignment of the prior. But it does mean that after reasoned argument, a contextually knowledgeable reviewer, for instance, will not reject the assignment ${ }^{7}$. Like any other aspect of the scientific specification, sensitivity to judgements should be assessed by consideration of sensitivity and robustness to scientifically reasonable variations of the specification.

\subsection{The likelihood function and error model}

The likelihood, $P\left(D \mid C_{M}\right)$ embodies the comparison of model predictions to new data, i.e. data that was not used in the specification of the prior $P\left(C_{M}\right)$. If there were no uncertainty in the system and an adequate amount of data to uniquely distinguish each model configuration, then $P\left(D \mid C_{M}\right)$ would simply take the value 1 for the correct $\left(C_{M}\right)$ model, and 0 for all other model configurations. It is the presence of uncertainty ${ }^{8}$ which necessitates non-trivial probability assessment.

This uncertainty is usually first broken down into at least two broad categories. The most familiar category is observational uncertainties accounting for discrepancies between observational data and the physical system. This includes measurement uncertainties, such as in measuring the elevation of a fossil mollusc shell, and uncertainties in the relationship between the measured value and quantity of interest. The latter are often referred to as proxy or indicative meaning uncertainties, an

\footnotetext{
${ }^{7}$ Defensibility and transparency distinguishes the Scientific Bayesian interpretation, achieved by considering the spectrum of defensible expert judgement within the field (Goldstein, 2006).

${ }^{8}$ The impossibility of exact inversion for non-trivial simulators also leads to this necessity.
} 
https://doi.org/10.5194/cp-2021-145

Preprint. Discussion started: 17 November 2021

(c) Author(s) 2021. CC BY 4.0 License.

(c) (i)

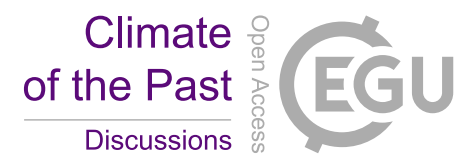

example of which is the difference between the present-day elevation of a fossil mollusc shell sample and past relative sea level.

The category that is often ignored is simulator uncertainties due to limitations of the simulator in representing "reality". This includes limitations in simulator structure, initialization, and inputs.

It is useful to further break down simulator uncertainties into those that are reducible (via an improved choice of simulator inputs and/or parameters), and those that are irreducible (for at least the near future, e.g., due to climate models not resolving individual clouds). The irreducible errors are often referred to as the structural uncertainty or structural discrepancy.

The relationship between observational and simulator errors, simulator prediction, and observations can be conceptually summarized as follows:

$$
\text { observation }=\text { Reality }+ \text { observational error }
$$

and on the simulator side:

$$
\text { Reality }=\text { simulator prediction }+ \text { simulator error }
$$

Substituting for Reality into the first word equation :

$$
\text { observation }=\text { simulator prediction }+ \text { simulator error }+ \text { observation error }
$$

rearranging $=>$

$$
\text { observation }- \text { simulator prediction }=\text { simulator error }+ \text { observational error }
$$

However, we do not know the actual errors, which would require apriori accurate identification of the state of reality. We therefore replace errors in the above relationships with a statistical error model for the distribution of these errors. For instance, the most common error model for the measurement error component of observational error is a Gaussian distribution with 0 mean (assuming the measurement device has been properly calibrated) and specified standard deviation.

The error model not only includes the chosen statistical distribution, but also the chosen dependencies. For measurement error, a common assumption is that the measurement error at one time and/or location is independent of the measurement error at another time and/or location. In actuality, most measurements, at least in a paleo context are likely to have some error correlation in both space and time. Example sources of this correlation include the local reference error for field measurements of sample elevation as well as sample preparation and mass-spectrometer calibration contributions to errors in radiocarbon age extraction. However, for most paleo contexts, inferential errors introduced from the assumption of measurement error independence are likely of relatively minor impact. More substantial correlations over space and time in the error structure are often introduced, for instance, in the age calibration of the sample from uncertainties in the $\mathrm{C} 14$ reservoir age for a marine region.

Depending on the measurement device, the measurement error may or may not be independent of the measured value. For the dependent case, this often occurs when the measurement uncertainty is specified to be a constant percentage of the measured value. In this case, a logarithmic transformation can still provide independence, which aids computational tractability. 
Converting our above conceptual equation between an observation and model prediction to a probabilistic statement, we then get:

P(observation - simulator prediction | simulator, configuration)

$=P($ observational error + simulator error $\mid$ simulator, configuration $)$.

Symbolically, with "R" referring to the inaccessible reality (e.g., ice sheet topography during last glacial maximum), and "M" referring to model simulator and associated simulator output, the above relation becomes algebraically self-evident:

$P\left(D-M\left(c_{M}\right) \mid M, C_{M}=c_{M}\right)=P\left((D-R)+\left(R-M\left(C_{M}\right)\right) \mid M, C_{M}=c_{M}\right)$,

with " $\left.\mid M, C_{M}=c_{M}\right)$ " indicating conditioning on the choice of model ( $M$, though usually this is only implicitly assumed) and the choice of model configuration $\left(c_{M}\right)$. The right-hand side of the above equation provides the tractable form for specifying the likelihood as a function of error models for both observations (i.e. $D-R$ residuals) and the simulator $\left(R-M\left(C_{M}\right)\right)$. As we are seeking a posterior distribution for the model configuration $\left(c_{M}\right)$ to have only irreducible structural errors, the likelihood also needs to be conditioned on the model configuration having no reducible error ${ }^{9}$. Therefore only the the irreducible structural error model is used for the simulator error in eq. 13.

Given that the above relationship (eq. 13) is conditioned on $C_{M}$ (i.e. predicated on $C_{M}$ having some specified value $c_{M}$ ), the left-hand side of the above equation then equates to the likelihood function $\left(P\left(D=d \mid M, C_{M}=c_{M}\right)\right)^{10}$ since:

$$
\begin{aligned}
P\left(D-M\left(c_{M}\right) \mid M, C_{M}=c_{M}\right) & =P\left(D-M\left(c_{M}\right)=d-M\left(c_{M}\right) \mid M, C_{M}=c_{M}\right) \\
& =P\left(D=d \mid M, C_{M}=c_{M}\right)
\end{aligned}
$$

The first line just expands what $P\left(D-M\left(c_{M}\right) \mid M, C_{M}=c_{M}\right)$ means while the second line uses the conditioning on $M\left(c_{M}\right)$, i.e. the probability distribution for $D$ will be determined by the distribution for $D-M\left(c_{M}\right)$ as $M\left(c_{M}\right)$ is a constant given the conditioning.

Consideration of the given definitions may aid intuitive understanding. The likelihood function computes the likelihood that the simulator output is consistent with the data. This consistency is specified by the error model, i.e. the uncertainty distribution for combined discrepancies between data and reality and simulator and reality (together this gives the distribution of discrepancies between data and simulator).

The simulator error model specifies the structural error distribution (i.e. the errors that are irreducible over the range of model configurations $\left(C_{M}\right)$ ), so that Bayes rule then provides the posterior distribution for the simulator configuration with irreducible structural error. Other configurations will tend to have lower posterior probabilities, usually (but not always) associated with larger overall values of data model residuals $\left(d-M\left(c_{M}\right)\right)$.

To make this more this concrete, consider a simple example of the likelihood for a single observational datum $(D=d)$ assuming that both observational and structural error models are Gaussian distributions with 0 means and respective variances $\sigma_{o b s}^{2}$ and $\sigma_{m o d}^{2}$. Also assume that they are both independent of each other and of the model configuration. Denoting the

\footnotetext{
${ }^{9}$ This conditioning requirement is consistent with the meaning of conditional probability, i.e. $P(A \mid B)$ is the probability of $A$ being true given that $B$ is true.

${ }^{10}$ The likelihood function should not be viewed as a conditional probability function because $D$ is held fixed while the input $c_{M}$ is varied.
} 
corresponding model output as $m\left(c_{M}\right)$, then the likelihood is

$$
\begin{aligned}
P\left(D=d \mid m\left(c_{M}\right)\right) & =P\left(d-m\left(c_{M}\right) \mid m\left(c_{M}\right)\right) \\
& =P\left(d-m\left(c_{M}\right)\right) \\
& =\operatorname{Gau}\left(d-m\left(c_{M}\right) ; 0, \sigma_{o b s}^{2}+\sigma_{\text {mod }}^{2}\right)
\end{aligned}
$$

where $\operatorname{Gau}(\cdot)$ is the Gaussian density function with specified mean (0 in the above) and variance. The second line above used the assumed independence of the error distributions on $c_{M}$. The third line used the standard result that the sum of two independent Gaussian distributions is still Gaussian with variance given by the sum of the individual variances and mean given by the sum of individual means.

In summary, the likelihood is fully specified by the chosen error model. A major challenge is specifying the structural discrepancy component of the error model. Within most (if not all) scientific disciplines, the tendency to date has been to effectively ignore this source of uncertainty. This can result in large inferential errors as we now show by example.

\subsection{A simple inference example illustrating the importance of accounting for structural discrepancy}

As perhaps the simplest illustration of the importance of addressing structural discrepancy, consider the following toy model. Let reality be the simple linear relationship $R(x)=20+5 x$ (red line in Fig. 2), and given that our models are always simpler than reality, let our model be $M(x)=C x$, i.e. our model approximation of reality is forced to a $0 \mathrm{y}$-intercept. If we ignore the structural error of a forced 0 y-intercept, and blindly carry out standard linear regression against the data (with additive Gaussian noise) indicated in Fig. 2, then $M(x)$ and its $2 \sigma$ confidence interval are the blue lines. The computed confidence intervals capture the relevant fraction of "observational" values, as expected for linear regression. However, the inferential value of this linear regression beyond the data range quickly becomes negligible. Not only does $M(x)$ diverge from $R(x)$ for larger $\mathrm{x}$, but even the standard $2 \sigma$ confidence interval fails to capture the true $\mathrm{R}(\mathrm{x})$ beyond the data range. Furthermore, an increase in the density of data-points limited to the indicated range of data will not improve the inference.

Given that models in the Earth Systems context are of interest for their predictions beyond the observational data range (across both space and time), this example shows the problematic consequences of ignoring structural discrepancy. Predictions from simulations that "best fit the data" can quickly diverge from reality and, more critically, estimated uncertainty bounds can significantly under-represent actual model-data differences.

Brynjarsdottir and OHagan (2014) offers a complete example of Bayesian calibration of a slightly more complicated toy model that quantitatively elucidates how the lack of accounting for structural discrepancy will bias inferences. In both their toy model and ours, it is also clear that when structural discrepancy is ignored although the addition of more data within the existing data range will tend to reduce posterior variance, it will not reduce the inferential error: "with more and more data ... we become more and more sure about the wrong value for" the model parameter (Brynjarsdottir and OHagan, 2014).

For less idealized examples that examine inferential errors when structural discrepancy is ignored or over-simplified we must turn to the literature in other fields. For the context of a conceptual rainfall-runoff model, Schoups and Vrugt (2010) consider a mis-specified error model that lumps together observational uncertainty and structural discrepancy into a Gaussian distribution 


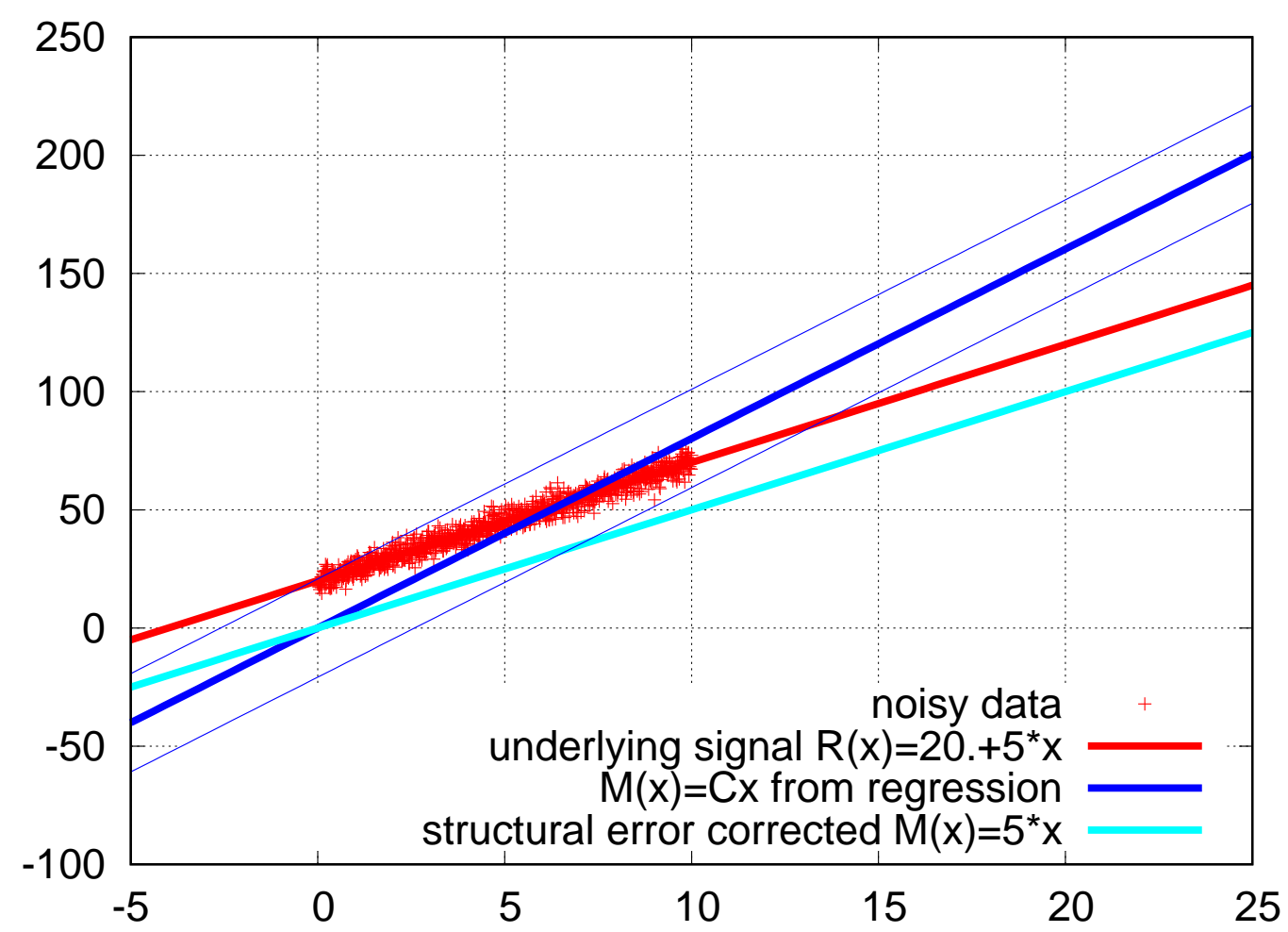

Figure 2. Linear regression example of inferential error that can occur when structural discrepancy is ignored. The red line is the underlying "reality" to which Gaussian noise is added to generate the 1000 sample data-points. The dark blue lines are the linear regression results (mean and $2 \sigma$ confidence interval) for the structural incomplete approximation with 0 y-intercept.

with 0 mean and constant standard deviation (a not uncommon practice). They show this results in a posterior distribution for model parameter vectors that has no overlap with those obtained when the error model is more appropriately specified. Tavassoli et al. (2004) also provides an applied example in the context of geological petroleum reservoir estimation with no structural uncertainty assessment of how the models that "best fit the data" can have "bad" inferential value.

Given the structural limitation imposed on our toy model, the useful inference would give the model $M(x)=5 x$. Though this model has a poorer fit to the observational data, its confidence interval captures $R(x)$ beyond the data range. As such, the model has clear predictive value.

Consideration of even this simple illustration raises the difficult challenge of how to account for the structural uncertainty. Conceptually, having access to "reality", we can easily see the appropriate choice for the optimal model $(M(x)=5 x)$, and therefore determine the structural error as a constant bias: $R(x)-M(x)=20$. However, for realistic contexts, we generally lack the omniscient perspective. Dropping omniscience for our toy example, as long we have confidence in our observational uncertainty (especially that observational uncertainties do not grow at the ends of the data range), one could test a range of values for the slope $\mathrm{C}$, and then note the simplification in the structure of the residuals as the test value approached the 


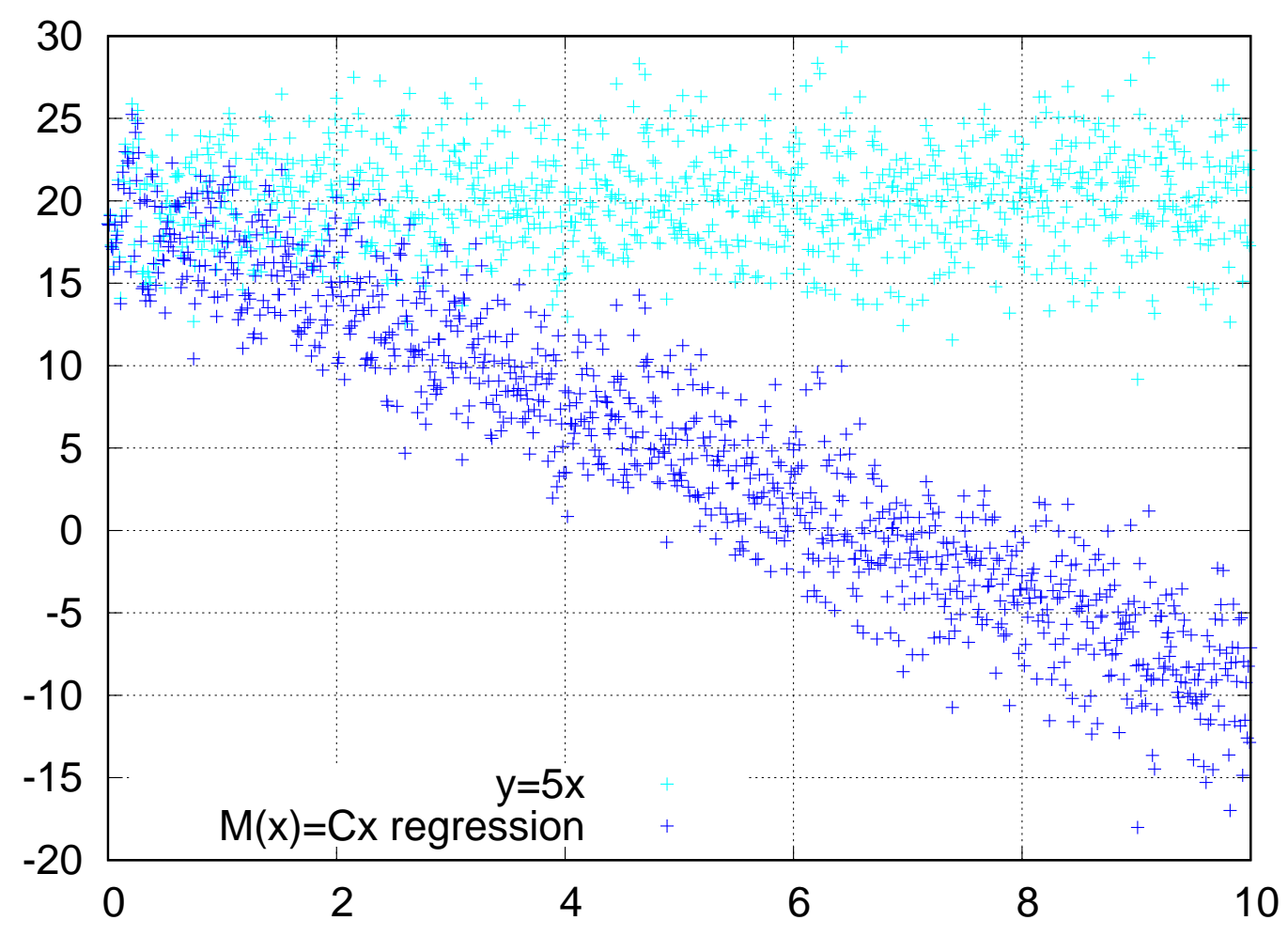

Figure 3. Data - model residuals for the toy model in Fig. 2.

actual value of the underlying reality ${ }^{11}$ (Fig. 3). But in considering models of earth system components in four (space-time) dimensions, such inference becomes much more difficult as the underlying residuals between model and reality will have a nontrivial multivariate structure (i.e. model-observations residuals from proximate observations being more strongly correlated) that will generally not correspond to any standard statistical distribution.

Further consideration of the toy example can illustrate the incorrect inference that can result from incorrect specification of the structural error. For instance, one not uncommon approach is taking the structural error to be some scaling (with scale factor greater than 1) of the minimum residual between simulator output and the observational dataset across an ensemble (e.g., , as in Annan and Hargreaves, 2002). This choice of structural discrepancy would still lead to the same erroneous selection of the model that minimizes the root mean square of the data-model residuals, i.e. that already obtained by linear regression $(M(x)$ in Fig. 2).

Given that our toy example is the simplest linear model, the much higher complexity and associated non-linearity of current models of geophysical systems such as ice sheets and climate makes inferences with such models potentially much more sensitive to inaccurate specification of structural discrepancy. Thus, unless structural discrepancy is accurately accounted for,

\footnotetext{
${ }^{11}$ It should be noted that the blind linear regression in this example would be rejected by standard statistical diagnostics or by simple inspection of the residuals shown in the figure.
} 
https://doi.org/10.5194/cp-2021-145

Preprint. Discussion started: 17 November 2021

(c) Author(s) 2021. CC BY 4.0 License.

(c) (i)

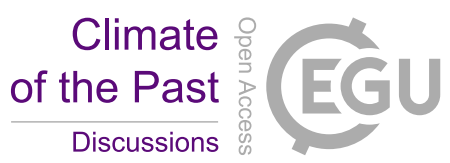

or at least not under-estimated in the model calibration, even studies that claim to use Bayesian inference may have much less retrodictive value and statistical confidence than indicated.

\subsection{Addressing structural discrepancy}

In order to address structural discrepancy, it is useful to first break it down into two components. Internal discrepancy is the component that is assessed by experiments with the simulator and external discrepancy is the remainder. Depending on context, we also separately differentiate potential internal discrepancy as the component of external discrepancy that could be assessed by tractable simulator experiments but to date have not been.

\subsubsection{Internal discrepancy}

Examples of internal discrepancy for paleo ice sheet models include the uncertainty in subglacial topography and deep geothermal heat flux for Antarctica and Greenland, uncertainty in initial conditions (such as the temperature field of the initial ice sheet, temperature field of the solid earth, initial isostatic disequilibrium, surface sediment distribution), and noise (randomness) in the climate forcing. For paleoclimate models, examples include: all initial conditions, ice sheet and topographic boundary conditions, atmospheric concentrations of aerosols and dust, and some of the contributions from unresolved turbulence (as much of the impact of turbulence won't be assessable by tractable simulator experiments). Explicitly stochastic components of a model representing such processes would also be contributors to internal discrepancy.

As a concrete example of an internal discrepancy determination for a single discrepancy source, consider the uncertainty of the subglacial topography under Antarctica and resultant impact on modelled glacial cycle evolution of Antarctica. After a pilot exploration to find some runs that don't obviously give bad fits to the data, one could extract a small, high-variance, and low collinearity (i.e. parameter vector directions well-separated) subset of approximately 10 parameter vectors. The associated simulations should also have high variance and low collinearity for values of metrics not directly constrained by paleo data (such as ice volume or mean annual precipitation over North America for some glacial time slice). The internal discrepancy determination would involve repeatedly re-running the simulator for each of the parameter vectors in this subset with different bed topographies. These topographies could be created by adding appropriately correlated noise to the available bed topography used in the original simulations. The correlated noise should be fitted to observed topographic variations in geologicallycorresponding regions that are free of ice cover after accounting for the sampling length scale of the observations used to create the original subglacial bed topography.

For each parameter vector, one could then extract appropriate statistics, such as the variance and covariance, of simulator output from the associated noise sub-ensemble of simulations with different topographic realizations ${ }^{12}$. These statistics would provide estimates for the statistical parameters in the probability density function for the internal discrepancy. For example, for each simulator parameter vector, one could extract the variance of present-day ice volume relative to the noise sub-ensemble

\footnotetext{
${ }^{12}$ For those statistically literate, in the case of a multivariate Gaussian error model, one would extract the variance/covariance matrix for each sub-ensemble. Matrix dimensions would include relevant simulator output for comparison against either observations (such as ice volume and basal temperature at ice core sites) or paleo proxies (such as ice extent at given time and location).
} 
mean. The maximum variance of each relevant simulator output across the set of noise sub-ensembles (i.e. one for each subset parameter vector) would provide an initial approximate estimate of the internal discrepancy variance of the simulator arising from topographic uncertainty. The stability of the assessed internal discrepancy could be assessed by examining how much present-day ice volume variance varied between the sub-ensembles. Hebeler et al. (2008) and Gasson et al. (2015) offer concrete examples of assessment of simulator sensitivity to topographic uncertainty for an ice sheet modelling context (though the assessment was limited to a few basic ice sheet characteristics and without consideration of covariance between different simulator outputs).

The set of variances and covariances from the set of noise sub-ensembles would be used to define a common variance/covariance representation for the given noise source (e.g., topographic uncertainty, basal drag uncertainty,...). This representation could then provide a multivariate Gaussian representation of the internal discrepancy. For spatio-temporal fields (such as temperature), the variance and covariance will need to include spatial and temporal dependencies. It may well be that the structure of the internal discrepancy is non-Gaussian with significant dependence on ensemble parameters. For both these cases, a more generalized statistical model is required to represent it (c.f. subsection 4.6 on emulation below).

Discrepancy from process uncertainties can also be partly assessed by similarly adding appropriate time-varying noise to, for instance, diffusion coefficients (to better represent turbulent mixing/transport) in climate models or to basal drag components in ice sheet models (to e.g., account for the impact of changing subglacial water pressure). If the strength or structure of this noise addition is subject to parametric control, then the parameter can be added to the simulator ensemble vector and this source of process uncertainty can be partly subsumed into the simulator ensemble parameter calibration. However, model sensitivity to the sampling of this noise (for a given parameter vector) will still need to be accounted for in the internal discrepancy assessment. This would entail repeated simulations with different initial seeds of the noise generator for the given process.

Internal discrepancy assessment is an important part of the simulator development process. Large sources of internal discrepancy point to processes that need to be explicitly incorporated into the simulator under ensemble parametric control. For our topographic glaciological example above, this might take the form of parametrizing the structure of the topographic noise, perhaps via mean amplitude and wavelength and/or variance parameters, and then adding these parameters to the ensemble parameter vector for the simulator. Prior ranges for these parameters would need to be consistent with available observational constraints.

415 For inferential contexts, one need only assess combined internal discrepancy for all considered sources at the same time. This will entail imposing all internal discrepancy noise sources simultaneously for each internal discrepancy sub-ensemble (i.e. using the same parameter vector). A concrete example of internal discrepancy assessment is provided in Goldstein et al. (2013).

To date, common practice implicitly assumes structural discrepancies are either minimal or, in the case of bias correction, relatively constant across different simulator states. These assumptions underly the common reasoning that a model that adequately fits past observations will have predictive value for the future. Internal discrepancy assessment can quantify the validity of such reasoning. 
https://doi.org/10.5194/cp-2021-145

Preprint. Discussion started: 17 November 2021

(c) Author(s) 2021. CC BY 4.0 License.

(c) (i)

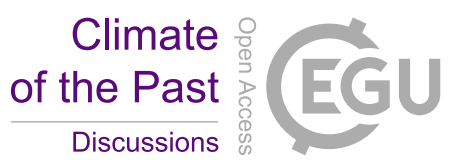

\subsubsection{External discrepancy}

The external structural discrepancy is the component of structural uncertainty not directly quantified by computer experiments.

Some common sources of external discrepancy are limited grid resolution of the simulator, parametrized (or ignored) subgrid processes, and uncertainties in inputs for paleo contexts. Determining an appropriate external structural discrepancy is one of the most challenging aspects of applying Bayes rule to the inference of past earth system evolution.

The previous toy example makes clear that the models that best fit the data within observational uncertainty can not be directly used to extract the structural discrepancy by setting the latter to the model-data residual less observational uncertainty. Bayes rule is predicated on the independence of the choice of the error model used to create the likelihood function from the model-data residuals input into the likelihood function. Concretely this means that initial internal and external discrepancy assessment must be done without consideration of model fits to observational data embodied in the likelihood. However, as discussed in the implementation section below, diagnostic testing can invalidate an error model and partially guide its subsequent re-specification.

The importance of structural discrepancy assessment can also be understood from the perspective of avoiding simulator over-fitting. The latter occurs when the predictive power of a model is sacrificed to increase model fits to data. Concretely, any set of $\mathrm{N}$ data points can be exactly fit by an $(N-1)$-th order polynomial. However, if the data contains any observational noise, then the regression is fitting reality plus noise, resulting in a loss of predictive power. This is especially clear when extrapolating beyond the data range given how fast high order polynomials can grow in magnitude as the independent input variable increases in magnitude. Bayesian model calibration would ensure model fitting to data is only within the context of a well-specified complete error model and thereby avoid over-fitting ${ }^{13}$.

There are a variety of complementary approaches towards addressing external structural discrepancy. They all start with assessing scientific understanding about sources of external discrepancy and making judgements about the resulting error for both prediction/retrodiction and likelihood comparison to constraint data. The judgements may be as simple as "given ice sheet model intercomparisons, dynamical sensitivities, a typical $20 \mathrm{~km}$ model grid resolution, and my own extensive experience as an ice sheet modeller, the fit to the observed grounding line positions for a model of the Antarctic ice sheet is likely to have a root mean squared error of at least $30 \mathrm{~km}$. Errors in grounding line position will in turn propagate into correlated errors in both past and present-day ice shelf areas." Each of these example considerations can then be backed up by appropriate referencing. The explicit specification of structural uncertainty enables reasoned evaluation in the review process and more broadly in the general community.

A second approach posits a multi-model ensemble (MME) of different state-of-the-art models as a closer representation of reality. A partial estimate for simulator external discrepancy can then be extracted from the distribution of residuals between each MME member and the closest member of a perturbed parameter ensemble (PPE), of the simulator under consideration. This requires a chosen metric to compute the distance between model runs. This approach would be of value if the structural discrepancy of the simulator of interest is significantly larger than the structural discrepancy of the MME. Sexton et al. (2011)

\footnotetext{
${ }^{13}$ For those with a machine learning understanding, one effect of the error model and prior is to collectively regularize the fitting of the simulator to the data.
} 
https://doi.org/10.5194/cp-2021-145

Preprint. Discussion started: 17 November 2021

(c) Author(s) 2021. CC BY 4.0 License.

(c)

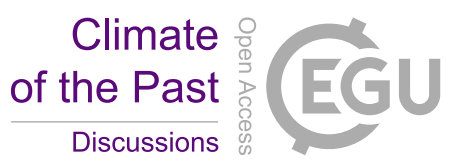

used this approach with a PPE for the HadSM3 climate model to generate probabilistic projections for the 21st century. They show (e.g., their figure 9) a significant impact on projected annual global mean temperature change if their choice of structural discrepancy is ignored.

A challenge in the use of such MMEs for discrepancy assessment is that available MMEs are generally ensembles of opportunity. As such, they incorporate models that are not independent (given the commonality of parameterizations, dynamical cores, grid resolution, and so forth) and to date are almost always only hand-tuned with no principled uncertainty assessment. All these factors limit the utility of current MMEs for structural discrepancy assessment.

One could also use a computationally expensive high-quality simulator to diagnose the structural uncertainty of a lowerquality, but computationally cheaper, simulator. Such an approach could be used to quantify a significant component of the external structural discrepancy contribution from an ice sheet model (ISM). It would require an ensemble of transient simulations of a high-quality ISM, such as a high resolution adaptive-mesh model with a higher order representation of ice flow (e.g., Cornford et al., 2013). Application of the same climate forcings to both the expensive high-quality ISM and the faster lower-quality ISM would permit extraction of a lower bound estimate for the lower-quality ISM external structural discrepancy. The completeness of this estimate would depend on the quality of components for processes at the physical boundary of the 470 ice sheet in the high-quality model.

It should be noted that if the high-quality simulator runs have yet to be done, there are much more efficient approaches for jointly using the information provided by a hierarchy of fast low-quality to expensive high-quality simulators (e.g., Cumming and Goldstein, 2009, as well as subsections below concerning emulation).

The fourth approach is to posit a parametrized form of the structural discrepancy and infer parametric coefficients. The posited form should be explicitly motivated by comparison with the results of other (hopefully structurally dis-similar) models (thus overlapping with approach three above) and/or physical reasoning and/or expert judgement. Even better if at least some of these other models in the comparison were of higher quality (i.e. one that has higher resolution or invokes fewer approximations at the cost of increased computational expense). The form of the structural uncertainty can further be constrained by underlying symmetries in the system under consideration (cf Arthern, 2015, and references therein).

The dividing line between internal and external discrepancy is in good part a choice by the modeller within the constraints of available time and computational resources. Discrepancies due to uncertainties in climate forcing for a paleo ice sheet model can in part be assessed by the addition of appropriate noise to the climate forcing. Such assessment would then convert a non-trivial fraction of the external discrepancy due to climate forcing uncertainties to an assessed internal discrepancy.

After careful consideration of structural discrepancy, the modeller may then judge it to be excessive for the given context. This would necessitate improvements to the simulator guided by consideration of the contributors to structural discrepancy. This involves some combination of : increasing simulator resolution, invoking fewer approximations in the simulator implementation of the relevant physics, and/or adding more parameterizations and associated calibration parameters.

To date, most may posit (as is often implicitly or, less frequently, explicitly done) the option of ignoring structural discrepancy and, for instance, just focus on finding the ice sheet chronology that most closely fits the observations within observational uncertainty only. One might then argue that the resultant chronology is that most consistent with data and physical relationships 
embodied in the employed simulator. If one's explicit purpose is to find such a chronology, in effect carry out simulator-based curve-fitting, this argument is unassailable. But what utility does this provide, especially for space-time locations where there are no high quality proximal observational constraints? If one is endeavouring to make an inference about the actual past and not about one's model, than the above toy model example should make clear how incorrect such an inference could be when structural discrepancy is ignored. For the surface and groundwater hydrology contexts, clear real-world examples have been published over a decade ago (e.g., Yang et al., 2007) showing the inferential errors that can ensue when structural discrepancy is ignored or under-specified.

\subsection{The normalization term of Bayes rule, Markov Chain Monte Carlo (MCMC) sampling, and emulation}

The normalization term, $\mathrm{P}(\mathrm{D})$, in Bayes rule need only be explicitly determined if absolute as opposed to relative probabilities are sought. To be a self-consistent probability, $\sum_{C_{M}} P\left(C_{M} \mid D\right)$ must $=1$. From Bayes rule, this in turn

$=\sum_{C_{M}} \frac{P\left(D \mid C_{M}\right) P\left(C_{M}\right)}{P(D)}$.

Inverting this equation leads to (aka the law of total probability):

$P(D)=\sum_{C_{M}} P\left(D \mid C_{M}\right) P\left(C_{M}\right)$

If one were only concerned with determining which paleo history (as defined by $C_{M}$ ) was more likely for a given ensemble of histories, one could ignore $P(D)$ and simply compare the product of the likelihood and prior for each history. However, inferring past Earth/climate system evolution entails determination of absolute probabilities and complete sampling of the nonnegligible posterior probability space of possible histories. This is where the computational challenge of Bayesian inference becomes apparent. $P(D)$ alone entails a sum of model evaluations over all parameter vectors $\left(C_{M}\right)$ and considered models (M) that have non-negligible prior probability and likelihood. For any model that can't be evaluated analytically, this sum must be approximated. For a single model system restricted to just 5 calibration parameters, even if we only considered a single parameter value from each decile of the prior, a simple factorial (grid) sampling would entail $10^{5}$ model runs. Especially when given an order of magnitude higher number of calibration parameters, this is clearly computationally unfeasible. Markov Chain Monte Carlo (MCMC) approaches (e.g., Andrieu et al., 2003; Richey, 2010, for accessible introductions) can significantly reduce this computational requirement via random walk searches through the parameter space, with the choice of walk steps weighted to the relative posterior probability of each trial parameter vector $(P(D)$ is not used).

The mechanics of MCMC sampling can be illustrated by a topographic example. Consider the problem of finding the lowest elevation point by in situ travel in a region of complex topography with sight-lines thereby restricted (i.e. given surrounding topographic highs). Suppose the only tool is a set of small transponders that can relay local elevation and an individual with a super-human throwing arm. This individual will randomly (with weighting according to perhaps a prior distribution for direction and distance) throw one transponder beyond his/her sight-lines (and usually beyond a proximal topographic high) and receive the local elevation of the point where the transponder lands. If the elevation is below the present elevation, the individual would then proceed to this new location and repeat the sequence. 
https://doi.org/10.5194/cp-2021-145

Preprint. Discussion started: 17 November 2021

(c) Author(s) 2021. CC BY 4.0 License.

(c) (i)

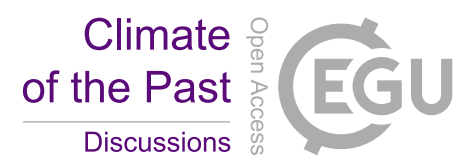

The crux of this illustration is the response to a resultant transponder elevation that is higher than the present position of the individual ( and therefore of lower analogous probability). If the individual were to consistently not go to regions of higher elevation, soon they would find themselves trapped in a local minimum (i.e. a valley deeper than adjacent valleys but not the deepest in the region).

To make this topographic search probabilistically self-consistent, or from a more limited optimization point of view, to search for the global minimum, higher elevation regions also need to be sampled. One approach (approximately corresponding to the Metropolis-Hastings algorithm) is to make the decision on whether to go to a higher elevation transponder location via a flip of a weighted coin. This weighting should be set to the relative probability of the transponder site being closer to the global minimum compared to the current location. Specification of this probability would properly need to take into account information such as the length scale of spatial correlation in elevation and surface slopes and the height of the transponder relative to the current position. For a transponder site with 50\% relative probability, this corresponds to flipping an unbiased coin, and only moving to that site if a heads results.

After convergence and thinning of samples to remove sequential correlations, the MCMC chains (sequence of sampled values such as measured elevations from the transponders in our analogy) will reflect the underlying posterior probability distribution. This means that values from high posterior probability regions will occur proportionally more frequently than values from low probability regions of the parameter vector space. This is a key difference from optimization algorithms that, for instance, will avoid repeated sampling of the same simulator parameter vector value. With MCMC, sampling from the full posterior may become more tractable. The actual number of sample points required will depend on the complexity of the model response to parameter variations or correspondingly the topographic complexity.

Continuing our topographic analogy, if one by chance starts in a high elevation plateau, it may take many transponder throws before one one ends up sampling the lowest elevation regions. This corresponds to the initial pre-convergence "burn-in" part of a MCMC chain. Scaling up our topographic analogy from the given two spatial dimensions to the order 30 to 100 ensemble parameter dimensions of typical paleo simulators makes the burn-in phase potentially much longer.

The rougher and more complex the topography, the longer it can take to exit a sub-optimal region. Such conditions also increase the sensitivity of the MCMC chain (search path) to the exact specification of the likelihood and starting point.

When dealing with high-dimensional non-linear systems (such as earth and climate systems), just as one can never be confident that a global optimum has been found, so one can never be sure if an MCMC chain has converged. To address this challenge in part, any study using MCMC methods should use some combination of standard MCMC convergence metrics and multiple (preferably hundreds of) MCMC sampling chains, each started from a dispersed sample of ensemble vectors from the prior.

In the first author's own experience with ice sheet model calibration of approximately 40 ensemble parameters, at least order ten million point sampling is still required (as compared to the astronomical $10^{40}$ for a simple grid search over deciles). As this is still beyond computational tractability for ice sheet and climate models, one other component is required. This component, a set of emulators, consists of very fast approximate statistical models that predict statistical characteristics of simulator output of interest as a function of an input parameter vector. 
https://doi.org/10.5194/cp-2021-145

Preprint. Discussion started: 17 November 2021

(c) Author(s) 2021. CC BY 4.0 License.

(c) (i)

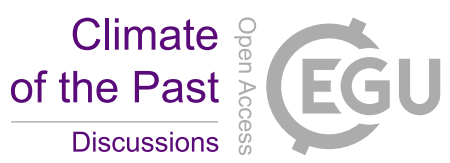

A single emulator might just predict a probability distribution for simulator ice volume as a function of the parameter vector and time. Or it might incorporate latitude and longitude inputs to predict simulator deglaciation times at specified grid cells. Thus, a set of emulators could collectively predict model output required for likelihood evaluation. To maintain statistical integrity of the inference process, emulators, by definition, must embody a probabilistic distribution for simulator output and therefore also compute and output their predictive uncertainty.

A more technical discussion about emulation is provided in the implementation section below. Any study claiming to infer meaningful bounds on ice sheet or climate system evolution using relevant simulators will require emulators to at least address parameter uncertainty.

\subsection{Uncertainty in making predictions/retrodictions: a missing term in most studies}

Once a climate or ice sheet model has been calibrated, or an ensemble of simulations has been otherwise generated, the ultimate goal is to make prediction/retrodictions and/or improve process understanding. The determination of a collection of simulator configurations consistent with constraint data after accounting for structural and observational uncertainties is only a first (though very large) step towards making a prediction. Consistent with the definition of structural uncertainty, predictions need to explicitly incorporate structural discrepancy. In our toy example, this would trivially entail adding a bias correction of 20 to the simulator prediction (light blue line in Fig. 2) to offset the structural error and recover the underlying signal (red line in Fig. 2).

Translating this toy reasoning into a Bayesian framework, one would seek the posterior probability for a future or past potential system state $S$ (or characteristic thereof, such as mean temperature) to equal value $s$ given constraint data $D$ and a simulator $M$ (or set thereof, by simply replacing $M$ with $\{M\}$ in what follows). Formally, this is denoted by:

$P(S=s \mid D, M)$

Invoking the standard statistical procedure of marginalization over all possible model configurations $\left(C_{M}\right)$, this

$=\int P\left(S=s, C_{M} \mid D, M\right) d C_{M}$

Marginalization follows from the basic axioms of probability. It corresponds to the reasoning that $P(S=s)$, i.e. the probability of $S=s$ irrespective of the value of any other quantities (such as $C_{M}$ ), equals the sum of joint probabilities of $S=s$ and $C_{M}$, $P\left(S=s, C_{M}\right)$, over the complete set of mutually exclusive values of $C_{M}$. Application of the multiplication rule to eq. 21 then provides a tractable expression in terms of the posterior distribution for simulator configurations $\left(P\left(C_{M} \mid D, M\right)\right.$, i.e. from Bayes Rule, eq. 7) and the likelihood for $S,\left(P\left(S=s \mid C_{M}, D, M\right)\right)$ :

$P(S=s \mid D, M)=\int P\left(S=s \mid C_{M}, D, M\right) P\left(C_{M} \mid D, M\right) d C_{M}$

To make this more concrete, for a climate model, D might be the observed record of precipitation and temperature. $S$ would be a statistic for paleo or future temperatures or precipitation or any other climate system characteristic to be inferred from simulator output. This statistic is any summary function of climate systems fields that are of interest, such as : mean monthly 
https://doi.org/10.5194/cp-2021-145

Preprint. Discussion started: 17 November 2021

(c) Author(s) 2021. CC BY 4.0 License.

(c) (i)

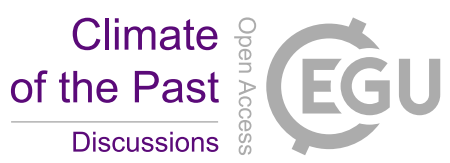

temperature, the exceedence or recurrence interval for some chosen extreme value threshold, yearly frequency of category 5 hurricanes,.. For a paleo ice sheet model, $S$ would include ice sheet thickness and surface elevation over each geographic position and time, while $\mathrm{D}$ is the collective set of constraints used for the ice sheet model calibration.

The likelihood $P\left(S=s \mid C_{M}, D, M\right)$ in the above equation makes explicit that any predictions must account for structural discrepancy in the relevant simulator output (and not just in the simulator output data constraints used to determine the posterior for $C_{M}$ ). As mentioned in the previous discussion on structural discrepancy assessment, the practice of bias correction is a simplified form of imposing the $P\left(S=s \mid C_{M}, D, M\right)$ term, by assuming that the total predictive error is a time-independent bias term (such as the difference between present-day reanalyzed temperature and simulated temperature from a climate model).

It should be noted, that the description to this point assumes a single best best input choice for $c_{M}$. A more general approach, especially when components are not physically well-defined, is presented in Sect. 3.1.

Aside from occasional bias correction, to date even most purportedly Bayesian studies with geophysical simulators ignore this crucial likelihood term and therefore underestimate predictive and retrodictive uncertainties. For those interested in implementation, Craig et al. (2001) offers a detailed example of Bayesian forecasting with a complex simulator.

\subsection{Multi-model ensembles and dealing with structurally different simulators}

For both paleo climate and paleo ice sheet (and sea level) contexts, there are a range of published simulations, many handtuned, some subject to ensemble-based scoring against limited sets of data constraints, and a few that attempt an approximate Bayesian inference. A natural question is how to make defensible joint inferences from such multi-model ensembles (MMEs) of simulations? This has received significant attention within the climate modelling community, in good part arguably driven by the challenge of drawing summary conclusions for International Panel on Climate Change (IPCC) contexts.

However, even after two decades of relevant literature and workshops, the topic is clouded by a preponderance of ad-hoc approaches and reasoning. This is evident in contrasting conclusions drawn from existing literature: "Owing to different model performances against observations and the lack of independence among models, there is now evidence that giving equal weight to each available model projection is sub-optimal" (Eyring et al., 2019) versus "an emerging body of research suggests that an unweighted average of all simulator outputs often performs favourably by comparison with performance-based weighting schemes" (Chandler, 2013). The latter came after a decade of literature considering different weighting schemes.

This confusion is most evident in approaches that take the ensemble mean and variance as the inferential best estimate and associated uncertainty. This choice presupposes that the ensemble members are random realizations of the physical system with independent, unbiased, additive errors. However for both climate and ice sheet contexts, simulator structural error will have dependence on the choice of simulator, invalidating this supposition. To make this clear, imagine if each MME member were a trivial climate simulator that output a different time-independent random temperature field. The MME average would have no meaningful relationship to the actual climate, contrary to the logic underlying the use of MME means and variances.

As indicated above, many approaches to MMEs assign weights to different simulations according to some performance score against historical observations without accounting for structural discrepancy. As in our toy model example, mis-specified weights (and therefore mis-specified or ignored structural uncertainty) will result in potentially highly inaccurate predictions. 
https://doi.org/10.5194/cp-2021-145

Preprint. Discussion started: 17 November 2021

(c) Author(s) 2021. CC BY 4.0 License.

(c) (i)

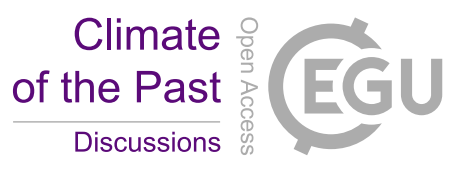

This has been borne out by explicit tests of performance-based weighting in climate simulation ensembles (e.g., Deque and Somot, 2010) as well as via detailed analyses with idealized simulators (e.g., Weigel et al., 2010).

The ice sheet modelling field has much less relevant literature on MMEs for paleo contexts. As one recent example, Batchelor et al. (2019) provide minimum and maximum extent time-slices for past Northern Hemispheric ice sheet evolution, in part via reliance on published numerical simulations. However, the set of chosen simulations do not include simulations designed to bound uncertainties in past evolution. A similar problem occurs in the climate field, for which simulator contributions to the IPCC are generally "best-tuned" versions, even though the IPCC attempts to infer bounds on future climate change from this under-dispersive set of climate simulations.

The previous subsection on making predictions with models showed how a posterior inference for any observable quantity requires two structural discrepancy contributions: one $\left(P\left(D \mid C_{M}, M\right)\right)$ to get the posterior for model parameters, and one for the prediction $\left(P\left(S=s \mid C_{M}, D, M\right)\right)$. Most MME weighting schemes ignore structural uncertainty in the likelihood (and therefore for the constraint data). Virtually all schemes to date, including "Bayesian Averaging", ignore the structural discrepancy for the final inferred quantity.

For those delving into MMEs, we recommend first the briefer but more accessible treatment (at least for most ice and climate modellers) of Williamson et al. (2013) followed by careful reading of Chandler (2013) and Rougier et al. (2013) which offer practical and theoretically rigorous methods for drawing inferences from MMEs.

\subsection{Constraint data : availability and uncertainty specification}

To date, based on the first author's experience with ice sheet modelling, assembling a relevant high-quality constraint data set with confident uncertainty specification has been a major challenge. Relatively recent (Briggs and Tarasov, 2013) and ongoing efforts such as HolSea (https://www.holsea.org/) have improved the situation, but what is really needed is a one stop shop for complete quality controlled constraint databases that includes detailed uncertainty specification. To be effective and efficient, such a data site will require easy and flexible data submission, error checking, automated quality control, automatic age calibration using a choice of protocols, bulk retrieval, and automated generation of a reference list for subsequent citation. In the context of relative sea level (RSL), Dusterhus et al. (2015) provides an overview of the issues to consider for such database design and implementation as well as a list of available RSL databases. Ghub (https://vhub.org/groups/ghub/paleodatasets) is a hopeful new endeavour that aims to fulfill many of these criteria for past and present ice sheets. Efforts by relevant communities to spell out data collection and logging protocols (e.g., Shennan et al., 2015) are also critical.

The ideal constraint data set will have narrow uncertainty and wide spatio-temporal coverage. It will also constrain diverse physical characteristics of the system. Consider the Greenland ice sheet. Present-day vertical velocities, relative sea level, and cosmogenic dates constrain earth model parameters for glacial isostasy as well as deglacial ice extent and peripheral ice thickness. The present-day observed (or reanalyzed) melt rate fields provide a strong constraint on modelled melt. Observed deep ice core temperature profiles provide an integrated constraint on past temperature and precipitation for central regions of the ice sheet. The present-day observed ice thickness field also provides a strong dynamically integrated constraint for glacial cycle models. 
https://doi.org/10.5194/cp-2021-145

Preprint. Discussion started: 17 November 2021

(c) Author(s) 2021. CC BY 4.0 License.

(c) (i)

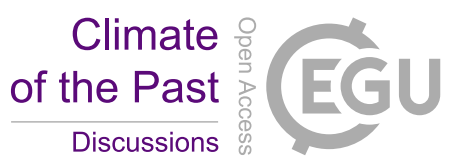

As discussed above, the specification of the likelihood (or an alternative to be discussed below) requires specification of constraint data uncertainty. In terms of the paleo context, the sea level and C14 dating communities have arguably made the most progress in data uncertainty specification. Shennan et al. (2015) provides detailed guidance in assessing RSL proxy uncertainties, especially in consideration of the relationship between the proxy (eg a mollusc found at a certain elevation, embedded in a specific stratigraphic framework, with a $\mathrm{C} 14$ date) and the "indicative meaning", i.e. the inferred probability distribution for the sea level associated with this datum. The dating communities are the most advanced with online applications that generate non-Gaussian probability distributions for calendar ages given a $\mathrm{C} 14$ or ${ }^{10} \mathrm{Be}$ datum. The provision of a computationally concise and efficient representation of these distributions (e.g., kernel density estimators) would facilitate their incorporation into paleo inference projects.

Error model specification for constraint data must account for the complete relationship between the model prediction and the datum. For the example of ${ }^{10} \mathrm{Be}$ dating, in addition to the uncertainties provided by online calculators, one must also account for uncertainties arising from inheritance and in the case of samples from boulders, transport and subsequent possible disturbance. For the case of minimum limiting C14 dates from samples embedded in an end morraine, the inference of when an end moraine was formed should include a distribution for time of migration of relevant flora to the sample site. All data/proxy comparisons must also account for uncertainties arising from limited simulator grid resolution.

Accurate observational uncertainty assessment can be challenging for poor-quality data. In noisy data such as RSL, it is common for much of the data to have no constraint value beyond increasing the confidence in other data. For instance, most dated mollusc samples (as in Fig. 4) only indicate that sea level was above the relevant datum for a given sample (with allowance for downward uncertainty of order 1 to 10 meters for possible tidal changes and upward displacement of the mollusc by waves). As such, only the highest mollusc samples for a given time offer useful constraint. In the example of Fig. 4, the lower-bounding, pre-3 ka data-points below 30 masl have no constraint value and should be eliminated from the constraint dataset.

This example illustrates the value of database cleaning. Clear outliers and data that apriori provide no additional constraint should be removed from the calibration (but should still be retained for subsequent posterior checks). Outlier removal requires careful, transparent, and documented judgement with explicit consideration of the whole set of local data and relevant signal. It is best done by those most familiar with the data and outlier assessment should therefore be a part of database entry by the data-gatherer.

\subsection{1 summary of what the Bayesian framework offers and challenges that ensue}

The above provides a high-level framework for making meaningful inferences about the world around us, especially in the context of incomplete models used to describe a complex non-linear world. It is also a framework for how to improve these inferences. For instance, attention to structural discrepancy can guide efficient simulator development by making clear the largest sources of structural uncertainty for the given context.

However implementation of standard Bayesian inference for complex simulators is a challenging and potentially non-robust endeavour. Posterior distributions tend to be non-analytical for non-trivial simulators and therefore can only be sampled from. 


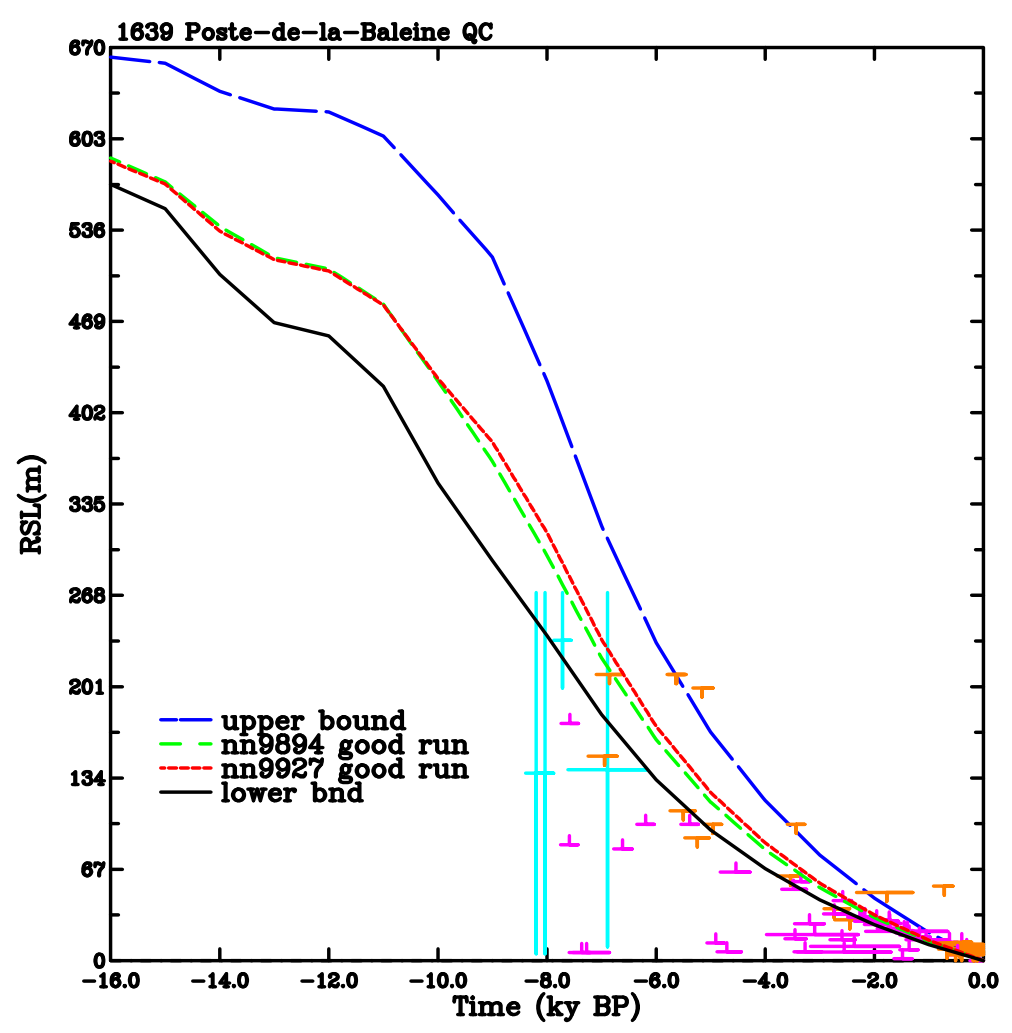

Figure 4. Comparison of modelled RSL histories and data for southwestern Hudson Bay Poste de la Baleine(Tarasov et al., 2012). To avoid clutter, 1-way error bars have been truncated. Orange 1-way (upper bound) error bars are generally for samples of terrestrial or mammalian origin, such as wood and bone. Purple 1-way (lower bound) error bars are generally non-intertidal species of molluscs that can live between shallows and depths of hundreds of meters.

As described in our discussion of MCMC sampling, this sampling may take a long time to converge (perhaps beyond available computational resource limits). No matter how the sampling is carried out, the result can be highly sensitive to the exact specification of the error model and therefore the likelihood. This is especially the case for high dimensional constraint data and parameter spaces, such as is generally the case for complex geophysical simulators. Our toy example also illustrates how inaccurate uncertainty accounting will lead to erroneous inference. For complex simulators, such error model specification will always be dependent on the uncertain specification of the external discrepancy. As such, inferences for say a most likely ice sheet history will have limited meaning contingent on a large set of assumptions. For those interested in a more complete and detailed accounting of the challenges involved, we refer the reader to the Frigg et al. (2015a) critique of the most detailed attempt at full Bayesian inference for future climate change to date (Sexton et al., 2011).

Unlike the norm to date, the Bayesian framework at least tends to force more of these assumptions to be explicitly stated and offers conceptual clarity. For many contexts, a more limited product than a rigorous posterior inference may have adequate 
https://doi.org/10.5194/cp-2021-145

Preprint. Discussion started: 17 November 2021

(c) Author(s) 2021. CC BY 4.0 License.

(c) (i)

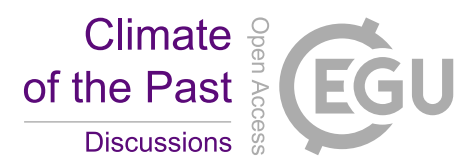

utility and can be much more robust. Such a product could also act as a useful stepping-stone towards a complete posterior inference. We turn to this now.

\section{Two stepping stones towards addressing uncertainty}

Below we describe two stepping stones that meaningfully address uncertainty within a tractable framework. Instead of a full posterior distribution, they collectively provide an initial set of uncertainty bounds and a sample of simulations within those bounds. Such bounds help reframe the emphasis of the inferential process from finding the most likely system history to that of inferring credible bounds on said history. Both stepping stones also align with a major pre-occupation of many modellers: evaluating to what extent one's model has the capability to capture the system of interest to the requisite minimal accuracy, identifying the components most in need of improvement, and then using this evaluation to improve one's model.

\subsection{The first stepping stone : History Matching}

History matching is a Bayesian approach that sidesteps the challenging requirements of a well-defined likelihood function and complete sampling from the posterior. Instead, it assesses whether individual simulator runs are implausible as approximate representations of reality. It is much easier to identify clearly bad simulator runs than to assign a defensible likelihood to all simulator runs. Furthermore, history matching can reject a simulator by assessing that all possible simulator runs are implausible. A full Bayesian analysis is predicated on the existence of one true value of the simulator configuration and therefore can't directly reject a simulator (however, posterior diagnostic validation offers an indirect route to rejection, $c . f$. subsection 4.5 below).

History matching was originally successfully applied to the determination of parameter settings for computationally expensive geological oil reservoir models (Craig et al., 1995; Cumming and Goldstein, 2009). It has subsequently been applied to simulators in a variety scientific contexts, such as ice sheet models (McNeall et al., 2013, though more as a proof of concept with limited metrics and only 5 ensemble parameters) and a general circulation climate model (Williamson et al., 2013). A complete application of history matching to a simulator for galaxy formation (Vernon et al., 2010) and to HIV transmission modelling (Andrianakis et al., 2017) in combination with an application of history matching to the NEMO ocean model that includes relevant code (Williamson et al., 2017) offers a clear presentation for those interested in implementation.

History matching involves a series of iterations ("waves") that, through an appropriate rejection criterion, incrementally refines the subspace of not implausible parameter vectors. A vector is deemed implausible if the output of the simulator or emulator is sufficiently far away from the observed data given all relevant uncertainties. This requires the definition of a set of implausibility metrics which quantify data-model misfits (residuals) relative to specified approximate values for total uncertainty. The simplest implausibility measure is the ratio of the data-model residual to uncertainty specified as a standard deviation:

$I=\frac{\text { model prediction }- \text { observed value }}{\sigma_{\text {total }}}$. 
Given that the square of total standard deviation for independent sources equals the sum of squared standard deviations for each source, it is more convenient to work with the square of the above equation. Furthermore, as adequate sampling of the parameter vectors will generally require emulators, "model prediction" in the above should be replaced by the mean emulator prediction for the simulator. Assuming respective emulator, structural, and observational standard deviations $\left(\sigma_{\text {em }}, \sigma_{\text {struct }}\right.$, and $\sigma_{o b s}$ ) for datum $d_{i}$ at location and time $x$, the implausibility value for the emulator mean prediction (technically expectation, $E())$ of simulator output $E\left(M_{i}(x, \mathrm{~cm})\right)$ using parameter vector $\mathrm{cm}$ then takes the form:

$I_{i}^{2}(x, c m)=\frac{\left(E\left(M_{i}(x, c m)\right)-d_{i}(x)\right)^{2}}{\sigma_{e m}^{2}+\sigma_{s t r u c t}^{2}+\sigma_{o b s}^{2}}$

740 A choice for an initial collective implausibility measure $\left(I_{M}\right)$ that avoids dealing with correlations in the error model is the maximum implausibility over the set of chosen constraint data $\left(I_{M}^{2}=\max _{i}\left(I_{i}^{2}\right)\right)$. Simulator runs with $I_{M}$ above a rejection threshold would be deemed implausible and ruled out. As $I_{M}$ would be very sensitive to inaccurate emulation of one single output for any simulation, it is generally advisable to set $I_{M}$ to the second or third largest implausibility $I_{i}^{2}(x, \mathrm{~cm})$ for at least early waves.

A common $I_{M}$ rejection threshold is a value of 3 based on the $3 \sigma$ rule (Pukelsheim, 1994) that at least $89 \%$ of a general probabilistic distribution should fall within 3 standard deviations of the mean. For any continuous unimodal distribution, this rejection threshold is more palatable as $95 \%$ of the probability will then fall within 3 standard deviations of the mean according to the Vysochanskij-Petunin inequality (Vysochanskii D. F., 1980). If it can't be ruled out that the underlying distribution is multi-modal, then a threshold of 5 would cover $96 \%$ of the probability for any continuous probability distribution (of an integrable random variable) according to Chebyshev's inequality (Chebyshev, 1867).

If the chosen threshold precludes an adequate number of not-implausible runs, then re-assessment of simulator configuration, inputs, choice of ensemble parameters, and/or simulator discrepancy distribution is required. This contrasts with a full Bayesian approach that requires the posterior probability distribution to integrate to 1 over all possible choices of the ensemble parameter vectors and therefore can't reject a chosen set of simulator, likelihood, and prior.

History matching is generally carried out with emulators, to ensure that the structure of the model response to the parameter space is reasonably well resolved. In our topographic MCMC analogy, "well resolved" would mean that every valley has at least one sample, even though the minima are generally not identified. During each successive wave, additional points in the parameter space are ruled out as implausible, leading to an ongoing refinement of the remaining not-ruled-out-yet (NROY) subspace.

History matching is much simpler to credibly implement than a full application of Bayes rule. It features a much freer selection of metrics and the lack of need for a multivariate error model and associated likelihood function, especially during initial waves. For instance, in calibrating a model for Greenland ice sheet deglaciation, one could initially separately apply RSL, present-day topographic, and ice temperature profile misfit metrics instead of a complete multivariate likelihood. A rigorous Bayesian inference would require the latter. As the NROY parameter space is narrowed through successive waves, internal discrepancy assessment can be carried out to refine the implausibility metric, including the addition of multivariate structure. 
Another useful feature of history matching is that there is no need to include all metric components for the initial waves. Instead, it makes more sense to start only with metrics that use easier to emulate outputs of the simulator. Furthermore, the emulators only need to be accurate enough to significantly shrink the NROY parameter space during the given wave. As the NROY parameter space is narrowed, accurate emulation becomes easier, especially for variables with spatial and/or temporal dependence. Further metric components can then be added. This improvement in emulator accuracy is due both to the narrowed range of simulator response and the increasing density of simulator runs in the NROY parameter space with each successive wave that is used to create the revised emulators.

History matching also offers an heuristic route to inferring an order of magnitude estimate of external structural discrepancy. Goldstein et al. (2013) provide an example of history matching using a fast rainfall runoff model with 17 ensemble parameters. In their case, out of 100,000 emulated model runs, they first select the best 8 that have the lowest implausibility when structural discrepancy is not counted (i.e. excluded from the denominator of equation 24). They then set the structural discrepancy for each metric component $i\left(\sigma_{\text {struct }}\right)$ so that the value of the full implausibility metric is just below the rejection criterion:

$\max _{\text {over } 8 \text { models }}\left(I_{i}^{2}\left(x, c m, \sigma_{\text {struct }}\right)\right) \leq 3$.

Though crude, such an estimate for structural uncertainty can be used as a starting point to assess whether the current model configuration is of requisite accuracy for the given context.

History matching is a learning process about the relationship between the simulator and the system in question. One does not require a complete and accurate error model for the first wave, only an error model that doesn't underestimate structural and observational uncertainties to one's best judgement. To avoid such underestimation, one can go as far as specifying a large structural discrepancy such that any larger would lead one to judge the simulator as useless for the given context. As one progresses through the waves, the error model can be refined. This thereby enables both learning about the structural discrepancy and the impact thereof on simulator calibration and predictions.

A methodological error to avoid is to initially underestimate the structural uncertainty and subsequently require its expansion. This would require a complete repetition of all previous waves. Such an error is much more difficult to avoid in a full Bayesian analysis. Given this and the requirement of a single accurate multivariate emulator for all relevant outputs that a full Bayesian inference would entail, we strongly recommend initial history matching even for those seeking a complete posterior distribution.

Note that a low value of the implausibility does not imply a plausible or "good" parameter vector, only that the vector has not yet been ruled out. A low implausibility of a parameter vector can arise from large emulator variance and/or lack of data constraints in the current wave for which simulator fits would be poor. For those interested in working through an actual toy example of history matching, Vernon et al. (2018) provide a detailed, lucid presentation (along with a non-toy case study for a systems biology model) that includes the complete R script in the supplement. 
https://doi.org/10.5194/cp-2021-145

Preprint. Discussion started: 17 November 2021

(c) Author(s) 2021. CC BY 4.0 License.

(c) (i)

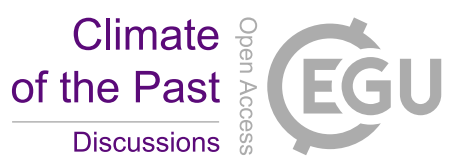

\subsection{The second stepping stone and structural discrepancy check: data-bracketing}

A pragmatic criterion that classical history matching doesn't explicitly address is whether the simulator has enough degrees of freedom to fully bracket (i.e. from both above and below) critical constraint data within observational uncertainties given parametric degrees of freedom. The choice of what constitutes "critical data" will depend on context (including correspondence of constraint data to quantities of interest for prediction), simulator complexity, and simulator cost. An NROY ensemble for which every member consistently overestimates present-day Greenland ice sheet ice thickness and ice area, for instance, would suggest some combination of too restrictive prior ranges for ensemble parameters and/or problems with inputs and/or some fundamental deficiency in the simulator. Such an ensemble would tend to have much less inferential value than one whose members collectively bracket all constraint data, preferably with bracketing by just two bounding simulations for each qualitatively different subset of data (such as temperature and precipitation for a climate simulator).

For computationally expensive climate simulators, the presence of persistent biases would necessitate a more restricted and context-dependent choice of critical constraint data that the ensemble would need to bracket. It will also require careful selection of ensemble parameter vectors informed by understanding of simulator response to individual and joint parameter variations. A persistent under-prediction of e.g., monsoon intensity may be unavoidable, and acceptable for say the context of coupling with ice sheet models for which tropical features have no direct relevance (though this could be complicated by teleconnections). However, for a similar context, a climate simulator ensemble for which all models under-predict the observed mean seasonal cycle over Northern Europe (a critical control on ice sheet surface mass-balance) would tend to have much less inferential value than one that fully bracketed the observed cycle.

From a statistical perspective, such bracketing would not be directly considered. Instead, any persistent biases would properly be subsumed into the (combined internal and external) structural discrepancy error model used to defined the likelihood. Such an allowance for simulator bias could therefore also be added to the implausibility metric in history matching. However, in nonlinear, spatially-temporally-coupled systems such as ice sheets and climate, large persistent biases are structural discrepancies that potentially make the resultant inferences of minimal value due to error amplification from positive feedbacks. This is especially the case for inferences related to simulator predictions not closely associated with available constraint data which is generally the case for paleo contexts. If the lack of data-bracketing were due to large external discrepancy (as opposed to internal discrepancy), this would induce a further inferential challenge of adequately specifying a large external discrepancy.

It is important to note that data-bracketing should not be a simulator tuning or calibration target as it doesn't account for structural discrepancy. It is also unlikely to hold for a final NROY set (given the lack of accounting for structural discrepancy). Instead, data-bracketing is a pragmatic early-stage modelling check on observational error models, parametric choices, and prior ranges for parameters. It may also force a reconsideration of whether the simulator structural discrepancy is acceptable for the given context. 


\subsection{An interim inferential reframing: bounding reality:}

Given the previously described challenges and fragility of full Bayesian inference, we urge a shift from the common focus on a best-guess chronology for the system under consideration to that of "bounding reality", i.e. determining credible upper and lower bounds on, for example, the glacial cycle evolution of an ice sheet. Even for very expensive simulators, such as general circulation climate models (GCMs), this would entail multiple simulator configurations designed to credibly offer some bound on key characteristics of the climate system that are of interest.

A full Bayesian calibration with a well-specified error model provides a posterior probability distribution for simulator predictions and therefore such bounds. However, for at least the near future, most modelling projects are unlikely to invest the resources for the required careful specification of the error model. Bounding reality offers a more accessible target. And history matching offers a tractable approach to reach this target.

An important part of bounding reality is ruling out that bounds are due to inadequate sampling of ensemble parameters, incomplete use of available data constraints, or inadequate structural discrepancy assessment. Ruling out of inadequate sampling requires use of validated emulators and an appropriate sampling scheme. The creation of relevant online community databases should help address data availability and assessment of the extent to which relevant paleo constraint data is used. Internal structural discrepancy assessment can be judged on the extent to which noise was introduced to all relevant processes and the appropriateness of the amplitude and structure of the introduced noise. The adequacy of external discrepancy assessment is more difficult to judge, though diagnostic checks described in subsequent sections can help.

A further check on the inferred bounds is from analysis of the contribution from data constraints and this ties in closely to data-bracketing. For instance, a number of studies using glaciological simulators for the last glacial cycle have inferred varying contributions to last glacial maximum sea level from Antarctica (e.g., Whitehouse et al., 2012; Golledge et al., 2014). However, to date, only Briggs et al. (2014) and Albrecht et al. (2020) have explicitly demonstrated that their glaciological simulators are able to produce significantly larger Antarctic contributions that were ruled out (tentatively in the first case) by data-constraints. These are also the two such studies that have inferred the largest upper bound estimates for Antarctica contribution to last glacial maximum sea level.

\section{Implementation and shortcuts}

The first parts of this perspective have outlined the general framework and challenges in carrying out a complete Bayesian inference for past ice and climate evolution. Rigorously carrying out such an inference can be a large-scale multi-year project beyond the ambitions of most modellers.

However, as we've argued above, uncertainty assessment is a critical part of the scientific endeavour and needs to be a core part of the whole modelling process. Just as in model building and experimental design, the researcher will need to make defensible judgements about trade-offs between inferential rigour and computational ease. Below, we sketch out a simpler framework for inferring past earth system evolution based on history matching. This framework provides a credible uncertainty 
https://doi.org/10.5194/cp-2021-145

Preprint. Discussion started: 17 November 2021

(c) Author(s) 2021. CC BY 4.0 License.

(c) (i)

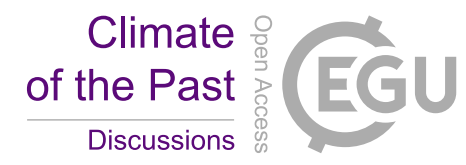

assessment as well as a stepping-stone to a more rigorous approach. We then selectively discuss implementation considerations for various components of the inferential process of relevance to both approximate and completely rigorous approaches.

All of this section, except for the next subsection 4.1, can be skipped by those not interested in the actual implementation of simulator-based inferences of past ice/climate/earth system evolution.

\subsection{A minimal framework for inferring past ice and climate system evolution with uncertainty quantification}

The following simplified framework offers an example approximate approach towards quantifying past system evolution. Furthermore, it would also form the stepping stone towards a complete Bayesian inversion. The example numbers of model runs given below are for ice sheet modelling contexts. For more expensive climate modelling contexts, run numbers can be reduced, in most steps, by an order of 10, with more attention put on efficient emulator development. Details on many of the steps are discussed in both prior and subsequent sections.

1. Assemble a team who can provide relevant expertise on: a) interpretation of paleo data and associated uncertainties, b) emulator development and uncertainty quantification, and c) proficiency and understanding of the simulator, including assessment of systemic simulator uncertainties and prior ranges for ensemble simulator parameters.

2. Select and/or develop a simulator relevant for the given context with both fast and computationally expensive higher quality configurations. The slow, higher-quality version of the simulator would include some combination of finer grid resolution and/or higher-order representation of relevant processes. The fast configuration should enable order one to ten thousand simulations. The exact number of runs will depend on the number of simulator parameters, choice of emulators, and complexity of simulator response. If the fast simulator has adequate accuracy for the given context, a slow version is not needed and the implementation framework can be appropriately simplified.

Relevant criteria for selection and development of both fast and slow simulators include the following. a) Process proximity to paleo constraint data (e.g., the use of relative sea level constraints requires a confident representation of glacio isostatic adjustment in the model system). b) Quality of the modelling, including : level of numerical validation, inclusion of processes judged to play a significant role, and defensible choice of approximations to the dynamical equations. These choices necessarily involve trade-offs, with a poorer quality simulator presumably being faster and therefore enabling more simulations, some of which may be required to address more sources of internal discrepancy. c) Ease/breadth of internal discrepancy assessment possible. For example, a model with multiple easy-to-implement options for basal drag (for an ice sheet model) or cloud representation (climate model) would enable a reduced estimate for contributions from harder-to-assess external discrepancy. d) Relatedness between fast and slow simulators to an extent that permits the fast simulator to be appropriately informative of the slow simulator. e) An adequate number of degrees of freedom in the forcing components (such as climate forcing for a paleo ice sheet model or volcanic forcing for a paleo climate model) to capture uncertainty in past forcing evolution on a scale relevant for the context.

For at least the paleo ice sheet modelling context, there are existing simulators available that can largely meet the above criteria with criteria (e) necessarily involving some trade-offs. This model selection step may require subsequent 
https://doi.org/10.5194/cp-2021-145

Preprint. Discussion started: 17 November 2021

(c) Author(s) 2021. CC BY 4.0 License.

(c) (i)

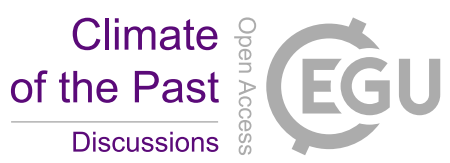

iterations to determine a fast simulator configuration (e.g., choice of resolution, and process approximations) that can be sufficiently informative of the expensive slow simulator response in the steps below.

3. Assemble paleo data. Selection should emphasize constraint value and spatio-temporal coverage (c.f. Sect. 2.10). Identify a provisional hierarchy of the data (c.f. Sect. 4.2) for history matching waves. Top-level data should provide wide spatio-temporal constraint. Consider what transformation of the data may be appropriate to facilitate implausibility metric specification ((c.f. Sect. 4.2 and 4.4). The choice of data hierarchy and transformation will need subsequent reconsideration according to which relevant simulator outputs can be accurately emulated during a given history matching wave.

Set aside a small hold-out subset of the data for assessment of the final NROY predictive/retrodictive confidence.

4. Identify potential ensemble parameters and specify their prior ranges. Start with listed simulator ensemble or tuning parameters. Identify the main sources of model uncertainty (such as basal drag and climate forcing in an ice sheet model). Introduce further ensemble parameters, as needed, to minimize the model uncertainties not covered by existing ensemble parameters (c.f. subSect. 4.3 below).

5. Run an initial order $\mathbf{2 0 0}$ member ensemble of the fast model with a dispersed random sample of parameter vectors from the prior ranges. Latin hypercube sampling (Urban and Fricker, 2010) is recommended as it ensures that the final set of vectors has values for every individual ensemble parameter well-spaced across its entire prior range. The ensemble should have variations in all simulator parameters that are not physically well constrained and that do not result in effectively duplicate simulator response.

6. Examine whether the model can bracket critical data (cf previous Sect. 3.2 for selection of "critical data"). Assess whether all top level data are collectively bounded within observational uncertainties by the ensemble. If not, reconsider: priors for the model parameters, deficiencies in inputs (especially degrees of freedom in the climate forcing for paleo ice sheet models), missing processes that could be explicitly incorporated, existing processes that could be better represented, the possibility of data processing and/or code errors, and the observational uncertainty error model (which specifies the relation of the datum to the model output, c.f. Sect. 2.10). If none of these solve the deficiency, consider if the slow simulator provides adequate bounding for the problematic critical data.

Ideally this bounding is surface-wise so that only a few ensemble members are required to collectively bound key data. However, it may be the case that a significant fraction of the key data can only be bounded point-wise, i.e. each datum requiring distinct ensemble members.

From past experience, this is one of the most time-consuming steps for model development. It also is an informative step in developing understanding of requisite minimal processes and inputs for the given modelling context as well as serving as a partial diagnostic check on specified parameter ranges, model configuration, and observational uncertainties.

7. Increase initial simulator ensemble size as needed for emulator development. For last glacial cycle ice sheet modelling 
configuration of Bayesian artificial neural network emulators of requisite accuracy for wave 1 history matching. Those using Regression Stochastic Process emulators (RSPE) may find a few hundred simulations adequate and would start fast/slow simulator emulator development at this stage, for which case slow simulator runs will also be required. Such development would use the global structure of the fast simulator RSPE to inform the structure of the RSPE for the slow simulator. More details on emulator development are in subSect. 4.6.

8. Carry out initial internal discrepancy assessment. Select two to three runs that best fit data constraints while being substantially distinct (sufficiently different parameters and features in the output). Generate a 50 member internal discrepancy noise sub-ensemble with the fast simulator for each parameter vector from the above runs. The noise should incorporate as many potential sources of internal discrepancy as feasible. Extract the resultant estimates for bias and variance components of internal discrepancy from the sub-ensembles (c.f. subSect. 2.6.1).

This assessment should also consider how internal discrepancy from fast simulator experiment can be used to estimate internal discrepancy for the slow simulator. At least a few internal discrepancy simulations with the slow simulator will therefore be required. Inflate the assessed discrepancy by an ad hoc amount, e.g., 10\%, to account for the limited number of runs and reliance on the fast simulator. The inferential error from imposing too small an internal discrepancy is much worse than that arising from too large an estimate thereof.

9. Provisionally assess external discrepancies for the slow model (c.f. Sect. 2.6.2) and add these to the error model used in the implausibility metric. This will require informed judgement by one or more experts familiar with the model.

10. Refine simulator configuration if required. Evaluate if the internal discrepancy is unacceptably large. If so, one will need to identify the specific problematic sources of internal discrepancy. To determine the strength of internal discrepancy sources, generate noise ensembles with a single noise source each (such as climate forcing noise for paleo ice sheet modelling). Start with those sources judged likely to be the largest contributors to internal discrepancy. Use the two basis parameter vectors from the previous internal discrepancy step that provided the largest internal discrepancy estimates (i.e. variance of relevant simulator outputs across their respective noise sub-ensembles).

Consider reducing the largest contributions by the addition of new ensemble parameters (i.e. not those already removed in the ensemble parameter selection step above). This can take both deterministic or stochastic forms, such as a coefficient controlling the addition of physically motivated precipitation anomalies in the climate forcing of a paleo ice sheet model in a correspondingly deterministic or stochastic fashion. However, ensemble parameter addition comes at the cost of repeating a number of the steps above.

11. Construct a test ensemble for emulators. Randomly sample 100 or more parameter vectors from the prior range and run these with the fast simulator. If using RSPEs, also create a small test set of slow model simulations.

12. Construct a set of emulators from the initial ensemble and select those that validate on the test ensemble for history matching. The validation should verify that the distribution of emulator-simulator residuals is consistent both 
https://doi.org/10.5194/cp-2021-145

Preprint. Discussion started: 17 November 2021

(c) Author(s) 2021. CC BY 4.0 License.

(c) (i)

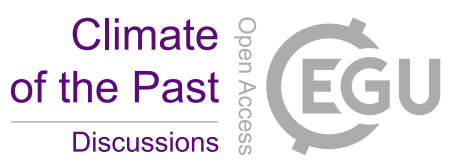

with the distribution predicted by the emulators and with any relevant assumptions that were made in the structure of the emulators. Emulators that fail this test can be rebuilt/retested during later waves with a refined NROY space.

13. Carry out an initial history matching wave with the emulators. The error model for history matching should include observational, internal and external discrepancy, and emulator uncertainty components. Uncertainty components should err on the side of over-estimation of possible uncertainty.

14. Refine NROY space with successive history matching waves. Preferentially choose parameter vectors for simulator runs from the NROY space with high emulator uncertainty. However, a few parameter vectors with low emulator uncertainty can provide a diagnostic check on emulator uncertainty self-estimation.

15. More carefully assess internal discrepancy. From the set of model runs, select 8 more runs with low correlation between key metrics and between associated parameter vectors that otherwise have the closest fits to the constraint data within observational and structural uncertainties. Use the cumulative 10 parameter vectors (including the two from the original assessment) to reassess internal discrepancy. In addition to internal discrepancy ensemble variance, consider biases and correlations. If there are significant correlations, then implement a multivariate implausibility. Consider which aspects of internal discrepancy can be emulated or transferred into a stochastic component of the simulator.

16. Construct an emulator for the slow simulator if not already done. Carry out 50-100 runs with the slow simulator using a dispersed NROY sample of the same parameters vectors already used for the fast simulator ensembles. Use the joint set of matched parameter slow and fast simulator runs to construct an emulator for the slow model. This emulator may be built on top of the existing fast emulator with the addition of emulation to predict the difference between slow and fast simulator runs for the same parameter vector. Both practitioner experience and some experimentation will facilitate effective emulator development for the slow simulator.

17. Carry out further history matching waves with expanding constraint data sets and/or refined emulators built on the cumulative set of simulator runs. History matching will be complete when the whole chosen constraint data set is imposed via the implausibility metric and emulator uncertainty has stopped decreasing. The mix of fast/slow simulator runs will depend on the choice of emulator, computational costs, parameter vector dimension, and accuracy of the slow emulators.

18. Reduce structural error as needed (and as is possible within the timeline of the project): Assess retrodictive/predictive confidence on the hold-out test data set (c.f. Sect. 4.5). Refine the simulator and add ensemble parameters to address persistent unacceptable misfits. Such refinements may necessitate reconsideration and revision of the structural error model.

19. The final NROY set of slow model runs, associated emulators, and associated structural uncertainties using the complete set of paleo data provides one's inferential estimate of past system evolution. This is an inference consistent with the simulator and constraint data, subject to the complete error model. This error model itself represents an 

important inferential product for making predictions/retrodictions (c.f. Sect. 2.8). Though a rigorous posterior probability distribution can not be defined without significantly more effort, the distribution of implausibility metric values has interpretative value as long the distinction between low implausibility and high probability is clearly conveyed.

Unless the NROY parameter space is very small, the set of simulations with this set will likely be an incomplete sample and therefore only provide a partial inference. As such, key predictions/retrodictions from the NROY set of simulations should be compared against predictions/retrodictions by emulators run over the whole NROY parameter vector set. Predictions for which emulator uncertainties are large can be further constrained by careful selection of further simulations to reduce emulator uncertainties.

20. Clearly state remaining misfits. This is of value to data gatherers to re-evaluate relevant constraint data and/or prioritize future data. The modelling community will also benefit from associated prioritization of required simulator improvements.

After a set of history matching waves that has reduced the NROY space adequately to allow appropriately accurate emulation of relevant simulator outputs, this minimal framework can be expanded in various ways. This could entail the specification of a full multivariate covariance structure for use in a Bayes Linear forecasting/retrocasting approach (Craig et al., 2001) or in sampling from the posterior (Andrianakis et al., 2015). The latter could also be carried out with an approximate, simplified likelihood as discussed below. Whatever approach is chosen, a posterior diagnostic checking of the error model (c.f. Sect. 4.5), and verification of MCMC convergence (if employed) should also be done.

\subsection{Selection of a hierarchy of constraint data}

The initial phase of history matching will use a limited high-quality subset of the data of wide spatio-temporal constraint value for which model response is easy to emulate. For instance, a relative sea level curve from Hudson Bay near the center of the Laurentide ice sheet will offer much more constraint and easier emulation than a deglaciation age from the northern periphery of the ice sheet. The latter would be quite sensitive to local ice load history and would only offer a spatially local constraint on past ice sheet evolution.

This hierarchical selection of constraint data can be guided by physical reasoning in conjunction with judgment of what are priority characteristics for the given context. Consider a climate model coupled to an ice sheet model for paleo contexts.

1015 First-order climate characteristics most relevant to ice sheet evolution would be mid- to high-latitude regional summer day-time temperatures (controlling surface melt) and yearly net snow accumulation. The strength of the seasonal cycle (summer minus winter temperatures) reflects sensitivity to insolation forcing and therefore would also be an important primary target for this context (assuming this sensitivity on seasonal scales has some relation to that on orbital timescales).

There are also rigorous options for hierarchical selection of data for simulator constraint. Cumming and Wooff (2007) and Cumming and Goldstein (2009) describe a relatively straightforward approach (principal variables) based on analysis of ensemble model output of variables that correspond to available data. They step-wise select variables that are most correlated with 
https://doi.org/10.5194/cp-2021-145

Preprint. Discussion started: 17 November 2021

(c) Author(s) 2021. CC BY 4.0 License.

(c) (i)

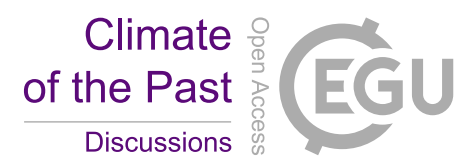

the remaining not-yet-selected variables after eliminating the effects of correlations already accounted for by the expanding set of selected variables. This is akin to step-wise variable selection in linear regression.

\subsection{Selection of ensemble parameters}

The choice of ensemble parameters should attempt to take into consideration all the possible parameters in the model. Geophysical models will have a set of specified explicit parameters, but there are a host of effectively implicit parameters for processes not explicitly parametrized or otherwise lacking appropriate parametric degrees of freedom. If one considers only the uncertainty in past climate over a glacial cycle, parametrized climate forcings for glacial cycle ice sheet models can easily add dozens of ensemble parameters. However most paleo ice sheet models use a handful or less of ensemble or tuning parameters for their climate forcing. For comparison, GCM climate models generally have more than one hundred poorly constrained explicit parameters. Even for simpler earth system models of intermediate complexity such as LoveClim (Goosse et al., 2010), there are at least a few dozen explicitly listed tunable parameters (Shi et al., 2019).

Ideally, the modeller will start with a large set of possible parameters, and then rigorously select a minimal subset that adequately probes the possible response of the model. This selection should encompass all parametric uncertainties, while minimizing duplicate response. For paleo ice sheet models, in addition to those from parametrized processes in the model (surface mass balance, sub-shelf melt, calving, basal drag, glacio-isostatic adjustment, and especially climate), consideration should be given to parametrizing uncertainties in ice sheet initialization and boundary conditions (such as the deep geothermal heat flux for all ice sheets as well as subglacial sediment cover and bed topography for Greenland and Antarctica).

The parameter selection process partly falls under the model sensitivity analysis rubric which quantifies model response to parametric variations. There is a large literature on this topic for approaches that at least partially account for interactions between parameters (e.g., Saltelli et al., 2008; Santner et al., 2003).

However a more direct, informative, and efficient approach for parameter selection is step-wise parameter selection for linear regression with linear, quadratic, and lowest order interaction terms (with the latter intelligently selected if simulator computational expense precludes an adequate number of runs). The regression should be repeated for all critical simulator outputs (or statistics thereof) on an initial ensemble with parameter vectors from an orthogonal Latin Hypercube (e.g., using the lhs R package) covering their full prior range. To improve confidence in the results, both step-wise delete and step-wise add selection should be run. Parameters that are not selected by any of the step-wise procedures would be tentatively rejected. This Latin hypercube will also be used for training emulators, thereby minimizing the number of simulations.

The initial reject set of parameters could then be tested by four more simulator evaluations that simultaneously adjusted all reject set members to their $1 \%$ or $99 \%$ prior range value, chosen so that all parameters were expected to give the same sign of simulator output response. The four simulations would have the non-reject part of the parameter vector set to two different (i.e. with large parameter space separation in distance and angle) basis vectors for which the simulator gave near best fits to data constraints. If this all at time subset test showed significant response, then a quick sort (Hoare, 1961) could be used to identify the subset of tentatively rejected parameters for which the simulator has a significant collective response. 
https://doi.org/10.5194/cp-2021-145

Preprint. Discussion started: 17 November 2021

(c) Author(s) 2021. CC BY 4.0 License.

(c) (i)

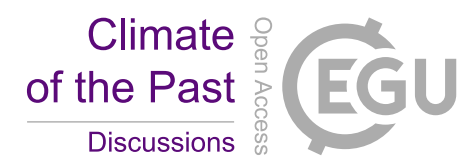

\subsection{Simplifying the error model: data transformation (dimensional reduction and aggregation) and data weighting}

Simulators of ice sheet evolution or paleoclimate will generally have correlated data-model residuals over possible ice sheet and/or climate trajectories. For instance, a simulator with chronically delayed deglaciation over northern Greenland will tend to have increased regional RSL and deglaciation-age data-model residuals. These correlations may arise between residuals of the same constraint data type (e.g., RSL or deglaciation age) but at different locations and/or times and between residuals of different data types. If this deglaciation delay was structural, then all these correlations would propagate into the structural discrepancy. As our above toy example has shown, accurate inference requires accurate accounting of structural uncertainty. Therefore, in general, structural discrepancy models need to account for this multivariate structure when sampling from a posterior.

The importance of accounting for such correlations for posterior inference can also be understood by considering the relative constraint value of different data. Consider, for instance, the relative sea level database for North America (Fig. 5). The largest spatial density of RSL data is over the Canadian High Arctic, a region covering only a few percent of the total LGM ice sheet extent. If each data-point were given equal weight and correlations were ignored, the much higher density of RSL data over the Canadian High Arctic would skew any sampling from a posterior toward models with better fits to this relatively small region at the likely cost of worse fits for the rest of the ice sheet. From a statistical point of view, equal weighting would imply that one is treating all the data-points as statistically independent, and thereby ignoring the high correlation between data-model residuals for RSL in this region arising from spatial proximity.

A multivariate likelihood function (such as a multivariate Gaussian) would account for all correlations between all available data-model residuals. Such a function would thereby, for example, not let the high RSL data-point density in the High Arctic skew a calibration. However, the robust assessment of multivariate structural discrepancy can be highly non-trivial and can become computationally expensive for large constraint datasets.

For at least all but final waves, history matching can avoid much of these challenges by not requiring a fully specified likelihood. This is because history matching only uses the error model in implausibility metrics for sample rejection (c.f. subSect. 3.1) and not for posterior inference. The metrics need only be based on those features of the joint structural discrepancy model that are easy to specify. Furthermore, especially for early waves, constraint data for history matching need only be a small highly informative (low uncertainty, high variation, and spatio-temporally dispersed) subset of what is available that is easy to emulate.

Even after database cleaning, for most geophysical contexts, the quantity of available constraint data can challenge likelihood specification and inferential tractability. As such, dimensional reduction of the constraint data space will often be required. The previously discussed use of principal variables (cf subSect. 4.2) is one option to consider for dimensional reduction. Principal components are another option that extract high-variance, dimensionally-reduced representations of the data. They are useful when there is extensive spatially-dependent data. They have been used to efficiently represent present-day grounding line position for a limited calibration of a glacial cycle model of the West Antarctic ice sheet (Chang et al., 2016). Sexton et al. (2011) similarly used principal component reduction in the context of GCM climate model calibration. Note, principal components 


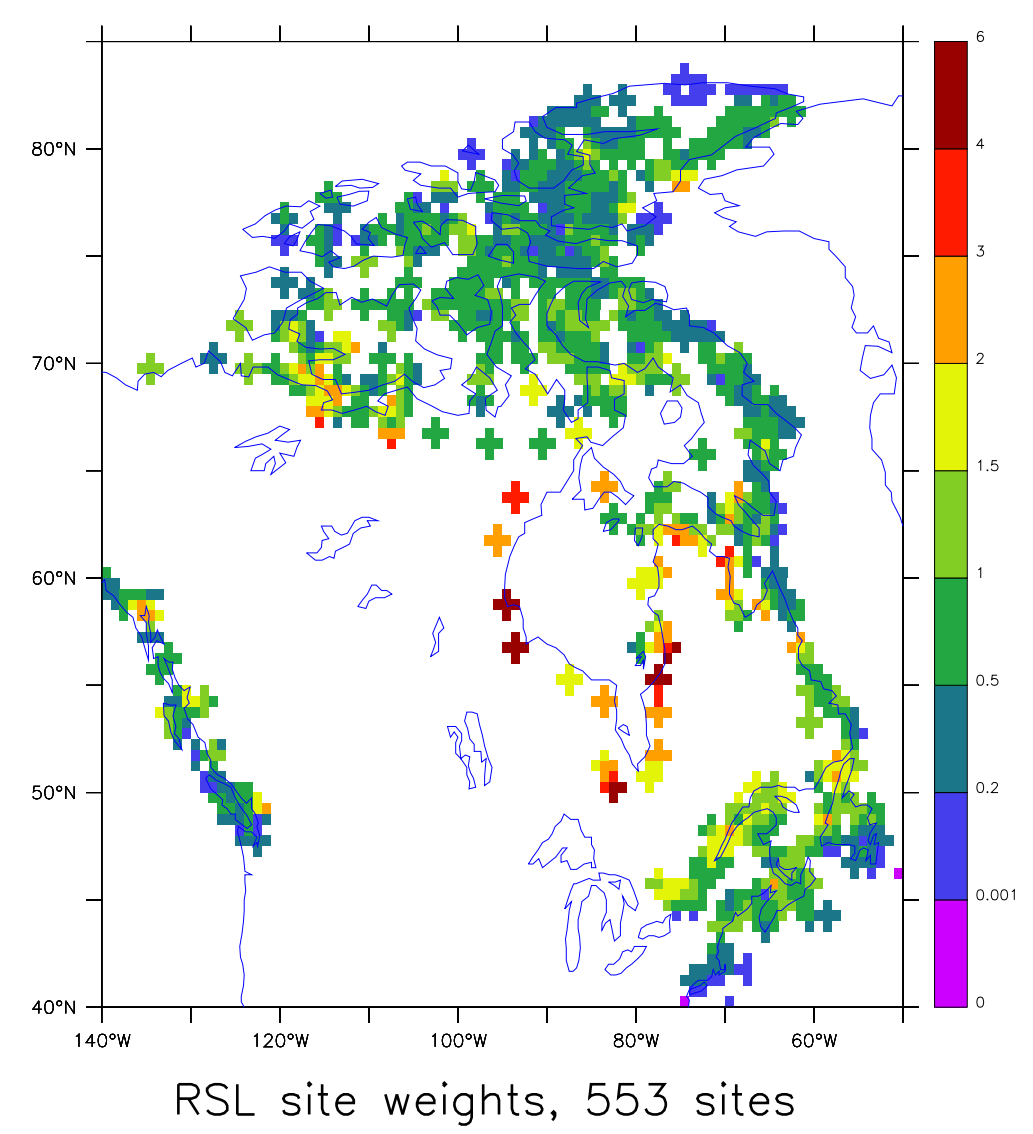

Figure 5. Specified site weights for a relative sea level database for North America().

can be noise sensitive due to a non-uniqueness problem for subsets of components corresponding to very similar eigenvalues.

They can also complicate functional dependencies, resulting in larger uncertainties for the emulator predictions and increased difficulty in specifying external discrepancy.

The community would benefit from an explicit comparison of options for dealing with large space-time dependent datasets, including: principal components, principal variables, and the addition of spatial dependence into emulators for different contexts. For instance, principal components are likely a good choice for global scale characteristics, especially when the components (or rotations thereof) are physically meaningful. Principal variables would tend to be better suited for characteristics with regional dependencies and scope.

The effective data dimension can also be reduced by spatial aggregation. Consider the collection of data-points aggregated to site Poste de La Baleine in Fig. 4. Instead of having the likelihood directly a function of each data-point residual, the whole set of residuals could be replaced by a single RSL site score. 
Unless all constraint data can be sensibly dimensionally reduced to a tractable size, both the computational costs of internal discrepancy assessment and the challenge of emulator development for a high dimensional multivariate likelihood may lead modellers to adhoc shortcuts that weight the data by some measure of effective constraint value (e.g., Briggs and Tarasov, 2013). However such weighting is a clear break from posterior assessment as the latter effectively measures simulation consistency with data given uncertainties and prior. This is logically distinct from measuring constraint value.

However a consistent synthesis is perhaps possible. The choice of constraint data for posterior inference or history matching requires informed judgement. One criterion is the constraint value of the data and data-weighting can be use to created dimensionally reduced representations of constraint data. For the RSL example above, this could take the extreme form of weighting RSL scores for all sites on an ice sheet to create a single RSL score. For which cases this is more appropriate than previously discussed approaches such as principal components and principal variables is a research question yet to be addressed. A significant challenge will be the specification of the likelihood as the observational error model will be convoluted into the aggregate data score.

Data-weighting is a heuristic approach and should therefore be explicitly justified. This could involve demonstrating that the inferential results have limited sensitivity to an appropriate range of plausible assumptions in the weighting scheme. Much better would be explicitly comparing results against a multivariate error model for a regional subset of the output space derived from internal discrepancy experiments.

\subsection{How to test structural discrepancy specification?}

Structural discrepancy specification requires scientifically informed and defensible judgement. Although internal discrepancy can be much more rigorously assessed (via simulator experiments), it is still subject to, for instance, the choice of noise forcing used in the assessment. As such, error models are always provisional. Furthermore, at least some of the assumptions that go into error model specification are likely to be broken for most geophysical modelling contexts.

If one is aiming to infer a posterior probability distribution, it is therefore important to assess whether inconsistencies between resultant high probability (or low implausibility) data-simulator residuals and error model assumptions significantly impact inferences for the given context. History matching is much less sensitive to the detailed structure of specified structural discrepancy and especially in early waves, one only need ensure that structural discrepancy has not been under-specified. A empty NROY space would be strong evidence of such under-specification. A very small NROY space may also be indicative of such under-specification, but it could also reflect inadequate sampling or very informative constraint data.

For the final history matching wave, a simple diagnostic check on error model specification is the comparison of NROY data-simulator residual distributions against the total error model. This comparison should be done for one simulation at a time over a small sub-ensemble that has the least implausibility score ${ }^{14}$. If then proceeding to a full Bayesian determination of the posterior, one would examine residuals for a sample from the final high posterior probability subset. This check is potentially

\footnotetext{
${ }^{14}$ Given the error model is only defined for the "most appropriate" parameter vector, one can't use a set of NROY simulations to create a sample as required for standard residual analysis approaches such as Q-Q plots. Instead, the data vector from an individual simulation could, for example, be transformed to a set of un-correlated standardized sample points, such as via an inverse Cholesky transform.
} 
https://doi.org/10.5194/cp-2021-145

Preprint. Discussion started: 17 November 2021

(c) Author(s) 2021. CC BY 4.0 License.

(c) (i)

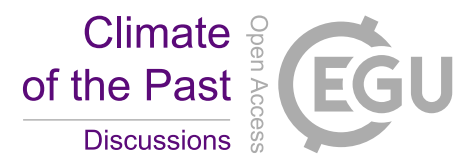

stronger if applied to a hold-out set of data not used in the history matching or posterior inference. For multivariate Gaussian error models, Bastos and OHagan (2009) offers a detailed example of such diagnostic checking.

The consistency between residuals and the error model is not a sufficient condition for error model validation. For instance, if the discrepancy between the observations + observational uncertainties and the blue linear (least squares) regression line in our toy model was chosen as the structural discrepancy, residuals would be consistent but the model would have much less predictive value than the optimal choice line after accounting for the biased structural uncertainty (light blue line in Fig. 2). This example reinforces the importance of making the selection of the structural error model (and therefore a component of the likelihood) independent of simulator fit to data. More fundamentally, the parametric form of the structural error and the prior for all simulator and error parameters needs to be set apriori, while parametric values of the structural error representation can be selected as part of the Bayesian inference.

Unlike history matching, full Bayesian inference assumes that there is a simulator configuration that is consistent with the likelihood, prior, and constraint data. It is therefore important to check for such consistency. Two relevant diagnostics for such checking are the prior and posterior predictive checks. Prior predictive checks are a useful diagnostic near the beginning of a Bayesian model calibration. They consider the prior predictive distribution, $P(d)$, for data constraint $(d)$ given a prior for the simulator parameter vector $\left(P\left(c_{M}\right)\right)$ and the likelihood function $\left(P\left(d \mid c_{M}\right)\right)$ :

$P(d)=\int P\left(d \mid c_{M}\right) P\left(c_{M}\right) d c_{M}$

Various diagnostics can then be constructed to examine the consistency of the predictive distribution with the actual datum $\widetilde{d}$. A cause for concern might arise if $\widetilde{d}$ were far in the tail of $P(d)$. As the above integral requires adequate sampling over $c_{M}$, it would need to be numerically evaluated with an appropriate emulator. Rougier (2007) provides a more detailed discussion of this diagnostic in the context of climate modelling.

Once a posterior for model parameters has been determined, the posterior predictive check (Kruschke, 2013) can be applied. It considers where the actual constraint data sits in the model posterior distribution for the data. This can thereby help diagnose the absence of any models that are consistent with the data. In contrast, history matching effectively checks the quality of the match between the model and data throughout the sequential waves.

Few detailed examples of diagnostic error model assessments for geophysical contexts have been published. In the context of a Bayesian calibration of a GCM, Sexton et al. (2011) and their followup Sexton and Murphy (2011) offer an example of some relevant tests that can be carried out for checking the order of magnitude of the structural error estimate as well as examining posterior sensitivity to assumptions in the error model. Schoups and Vrugt (2010) provide an example of how to assess a posteriori validity of the error model for a hydrological context which has the further complication of auto-correlation.

\subsection{Emulation}

Emulators are approximate models of simulators that explicitly estimate their own uncertainties relative to the predictions of the simulator. For our purposes, they can be thought of as generalized regression models. More accurately, emulators introduce 
https://doi.org/10.5194/cp-2021-145

Preprint. Discussion started: 17 November 2021

(c) Author(s) 2021. CC BY 4.0 License.

(c)

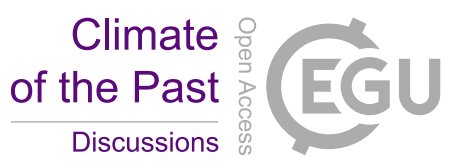

a probability distribution for simulator output as a function of the input parameter vector (with dependence on time and geographic location when appropriate).

Common emulators are regression models with Gaussian process residuals (e.g., OHagan, 2006; Rougier et al., 2009), or their second order equivalents which we'll denote as regression stochastic process emulators (relaxing the Gaussian process to a general second order stochastic process, e.g., Goldstein and Huntley, 2017), and, less frequently, Bayesian artificial neural networks (BANNs) (Neal, 1996). Given their greater familiarity, pure linear regression models (such as the $\operatorname{lm}($ ) function in R) can be used for initial waves (or depending on the desired reduction in the non-implausible space, can be used for a full history matching exercise, $c . f$. Ferreira et al., 2020).

The use of standard linear regression emulators has the major advantage of much wider familiarity within and outside of the statistical community. For initial waves in history matching, Ferreira et al. (2020) have demonstrated for a high dimensional (2136 outputs) synthetic case study of a geological water reservoir model that standard linear regression for second-order polynomial emulators can be quite effective. They achieved a $99.5 \%$ reduction in the parameter space after two waves. At this point, the reduction in the parameter space enabled adequate emulation of a multivariate objective function for the third wave (with only an increase of emulator complexity to third order polynomials, still with standard linear regression).

Regression stochastic process emulators (RSPEs) typically contain three terms. Firstly, a "global" (i.e. across the parameter space) term from linear regression with basis functions (such as polynomials) chosen with consideration of the expected response surface of the simulator. The second term is a stochastic process to characterize the residuals from the global term. This is a probability density function with explicit spatial, and possibly temporal, dependence, as well as dependence on the simulator parameter vector. This contrasts with linear regression that assumes residuals are independent and therefore will be especially more limited in emulating the simulator response to small variations of parameter vectors.

The last "nugget" term in an RSPE represents the remaining sources of discrepancy that do not show up in the residuals as a local white noise. This relates to a key feature of emulators with a linear regression global component for history matching contexts. Especially during initial waves, emulators of adequate accuracy for a given simulator target will tend to only require a small subset of the whole set of simulator ensemble parameters as inputs. RSPE's account for the impact of dropped parameters via the nugget. The active subset of inputs will vary between emulators for different targets. The potentially much smaller dimension of the active parameter vector simplifies emulator construction as well as parameter space sampling. For those interested in learning more about RSPEs, Vernon et al. (2018) includes a supplementary R script that reproduces RSPE construction and history matching for a simplified toy example.

A key advantage of emulators with regression and stochastic process components is that emulators developed for fast simulators can strongly inform the development of emulators for expensive slow simulators. This thereby minimizes the number of expensive simulator runs required for developing the emulator for the slow simulator. Rougier et al. (2009) and Williamson et al. (2012) offer two relevant detailed examples of such fast/slow emulator construction in the context of climate modelling. Cumming and Goldstein (2009) provide a more detailed derivation for such joint construction.

Emulation intersects with machine learning. Machine learning tools generally build computational models that generalize a training set of input/output relationships, such as in speech recognition. They could therefore also apply to advanced ice 
sheet and climate models. Machine learning (useful reviews include Ghahramani, 2015; LeCun et al., 2015) has undergone an explosive advance in capability over the last few decades as evidenced by the quality of speech and image recognition and successful application to highly complicated games such as Go. However, aside from the development of BANNs (e.g., Neal, 1996), the machine learning community has to date put much less emphasis on uncertainty estimation. Therefore many machine learning tools cannot be used as emulators unless an added structure is imposed. A second challenge is that machine learning generally relies on large training data sets, which might not be computationally feasible with complex simulators. The non-linear formulation of most machine learning algorithms also calls into question their ability to extrapolate as effectively required for the high dimensional parameter spaces of complex geophysical models.

As for any regression tool, emulators will tend to more accurately predict quantities that have a smoother and more systematic response to input variables. This can result in trade-offs between direct emulation of fields of interest (eg the ice thickness field over time for an ice sheet simulator) versus emulation of smoother quantities that can be more easily compared to certain observations such as relative sea level.

The development of emulators of requisite accuracy can be challenging and time-consuming, especially given a wide prior range of ensemble parameters values. An important shortcut within the history matching framework is to identify a subset of data constraints for which the relevant simulator predictions are easier to emulate (eg scalar values with smooth and more linear dependence on inputs such as present-day ice volume or global mean temperature). Initial history matching waves should be carried out with this subset. The resultant reduced NROY parameter subspace (and thereby reduced dynamical range of the simulator) can facilitate the sequential development of emulators for more difficult-to-emulate simulator outputs in subsequent waves.

An informative calibration requires validation that the emulator-predicted uncertainties are consistent with actual simulatoremulator discrepancies. Given sufficient simulator runs, this is most easily done with a set of parameter vectors not used to create the emulators. For a single output emulator with Gaussian uncertainty, this could be done by comparing the standardized residuals (simulator - emulator output expectation all divided by the predicted standard deviation of the emulator) to a standard Gaussian distribution. For expensive simulators, extra validation runs can be avoided with leave-one-out cross-validation. Bastos and OHagan (2009) provides a more detailed examination of emulator diagnostics that account for correlation between residuals.

Emulators are not just used to represent deterministic simulators. Emulators can also emulate models that have stochastic components. When internal discrepancy has spatial, temporal, and/or ensemble parametric dependence, an emulator can therefore offer an efficient representation. Such emulation also may enable the introduction of ensemble parameters (i.e. thereby subject to history matching and/or posterior inference) to set the structure or amplitude of the stochastic noise used to assess the internal discrepancy. For components of internal discrepancy that are relatively too large, this could thereby potentially reduce these contributions to internal discrepancy. 
https://doi.org/10.5194/cp-2021-145

Preprint. Discussion started: 17 November 2021

(c) Author(s) 2021. CC BY 4.0 License.

(c) (i)

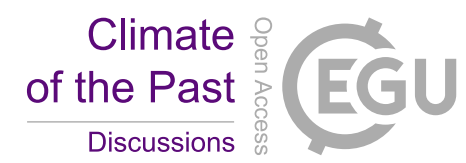

\section{Moving forward}

We offer below some key steps that relevant communities can take so that inferences about past ice sheet and/or climate states have meaningful value.

\subsection{Ensuring uncertainty is addressed in model-based studies}

Over the last two decades there has been an increasing rate of publication based on computer-based simulations of past earth and climate system evolution. Yet very few offer any clear uncertainty assessment. As such, the relationship between simulator output and system state is unclear. This becomes especially challenging for the non-modeller faced with often opposing inferences from simulation-based studies.

One core trade-off is between ensemble size and model resolution. How does one weigh the conflicting inferences of say a $5 \mathrm{~km}$ grid resolution hand-tuned model of Antarctica equilibrated for last glacial maximum (e.g., Golledge et al., 2012) versus a large (4000 member) ensemble of $40 \mathrm{~km}$ grid resolution last glacial cycle simulations with 31 ensemble parameters (Briggs et al., 2014)? More constructively, how do we intelligently synthesize the information from a handful of higher resolution simulations that lack uncertainty assessment with that provided by a much larger ensemble of lower resolution simulations that have been subject to some limited form of history matching and uncertainty assessment?

The above quandaries would strongly diminish if all model-based studies making inferences about states and state changes in past earth system evolution were required to have clear uncertainty assessments. Studies that focus on understanding the role of physical processes (e.g., through model sensitivity and/or feedback analyses) may not require data comparisons but should still explicitly consider to what extent their analyses are about the physical system versus just the simulator representation thereof.

A key step to towards encouraging rigorous uncertainty assessment about earth system evolution would be for modellers, editors, and reviewers to ensure the following questions are answered:

- What assumptions and approximations are made?

- What model parameters are adjusted and to what extent is the criteria used for their selection explicit and appropriate (c.f. Sect. 4.3)?

- To what extent does the chosen set of constraint data capture what is available and relevant?

- To what extent have the associated uncertainties been explicitly accounted for? In detail:

- What are the error models for the data and how have they been justified (c.f. Sect. 2.10)?

- How is structural discrepancy assessed for the numerical simulator (c.f. Sect. 2.6)?

- How has parametric uncertainty been addressed (c.f. Sect. 2.7 and Sect. 3.1)?

- Are predictive/retrodictive uncertainties appropriately assessed (c.f. Sect. 2.8)? 
https://doi.org/10.5194/cp-2021-145

Preprint. Discussion started: 17 November 2021

(c) Author(s) 2021. CC BY 4.0 License.

(c) (i)

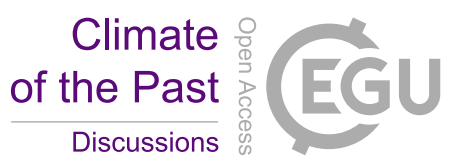

- How sensitive are the inferences to assumptions and approximations in the error model (c.f. Sect. 4.4)?

- Have relevant uncertainties been clearly communicated to the intended audiences (which usually will include those not familiar with the details of the model used)?

- Is an appropriate representative subset of the results of the higher likelihood or not-ruled-out-yet set of simulations provided from an open-access server?

As we've outlined in this survey, addressing all of the above are required if one is to make a principled approach to uncertainty assessment. Sufficiently addressing all points above is a major endeavour for any one or even a small group of researchers. Therefore a judgement has to be made and justified as to what are the most important aspects for the present context. At the very least, the answers to these questions should be explicitly provided in submissions that claim to make inferences about Earth system evolution to ensure that such inferences are scientifically interpretable.

\subsection{Addressing uncertainty in data-based studies}

As many data-oriented studies invoke models to relate measured quantities to inferred system characteristics, many of the considerations from the previous subsection are also relevant for them. Additional relevant questions for data-oriented studies include:

- Clear statement of assumptions in data interpretation.

- Clearer specification of errors than just \pm . Data providers need to consider a broad audience and detail uncertainties in the relation between proxy and inference. This should include an appropriate error model for the data that is specified or cited (c.f. Sect. 2.10).

- Emphasis on data quality. Though there is some trade-off, high quality data (i.e. with tight error bars and strong signal) is generally of much more value than a large quantity of low quality data (c.f. Sect. 4.4). Data gatherers can also use the uncertainty maps from calibrated modelling studies to prioritize data collection and ensure that their efforts will offer the greatest constraint value for modelling (c.f. Sect. 5.4). Tier rating of data quality in databases would also facilitate intelligent data usage.

- Deposition of data in centralized online databases. To ensure data-utilization, easy-to-use online data-servers are critical. Servers such as the world data centres (http://www.ncdc.noaa.gov/paleo/icgate.html) are a good example, but need to much more strongly encourage detailed uncertainty specifications, ease of conversion of data formats, and fully integrated age calibration tools (c.f. Sect. 2.10).

Those who gather, process, and make direct inferences from paleo proxies are most likely to have the clearest understanding of associated uncertainties. Data gatherers generally have their own conceptual uncertainty models that are only starting to be fully elucidated in peer-reviewed literature. An important contribution would be for each proxy community to develop 
a consensus error model for their specific proxies. By "consensus", we mean an error model that incorporates an extended distribution of all scientifically credible assessments of relevant uncertainties.

Data assessment and use requires easy data access in public online databases. Some journals and funding agencies are already implementing requirements to ensure such access. Perhaps the community can work towards entrenching this throughout the discipline. This requirement should not just apply upon publication, but also to unpublished data that has languished for say more than a PhD interval of 5 years since being gathered.

\subsection{A potentially new level of constraint : fully coupled ice and climate modelling of glacial cycle intervals}

As already evidenced in past climate model development, the coupling of previously separately forced dynamical earth system components, such as atmospheric and ocean general circulation models, can make the coupled system more unstable in response to mis-calibration and/or structural errors. Coupled systems, as such, can impose more self-constraint on simulator parameters and structure. The paleo ice and climate modelling communities are moving to a similar juncture with efforts towards fully coupled ice and climate modelling of extended glacial intervals including the complete last glacial cycle (c.f. https://www.palmod.de/).

To date, paleo ice sheet modellers have made extensive use of the large uncertainties in past climate evolution to provide easy tuning knobs for their glacial simulations. Fully coupled ice and climate models will have more limited tuning ranges as well as a wide collective set of constraints from both ice sheet relevant proxies as well as climate proxies. It may well be a major challenge for a fully coupled ice and climate model to just replicate the rapid last glacial inception inferred sea level lowering as well as the even more rapid sea level rise of the last deglaciation (Grant et al., 2012). Such replication would likely provide a strong constraint on model responses to radiative changes such as we are currently imposing on our planet. A tightening up of proxy-based inferences for sea level changes and rates of change during the last glacial cycle would therefore be very beneficial for such model constraint.

The coupling of two expensive simulators raises a number of interesting issues and questions for the context of this overview. If the two components have been previously history matched, how can the existing emulators and NROY sets be most efficiently used to facilitate history matching of the coupled simulator? How can the structural discrepancy of each component be explicitly introduced into the coupling? To what extent are internal discrepancies amplified due to feedbacks between the two components? Or are such amplifications more than offset by the parameter space restriction required for stability of the coupled model?

\subsection{Treasure maps: using inferential uncertainty to prioritize data collection}

The widely read book Sapiens (Harari, 2015) argues that the key reason for the success of the scientific revolution over the last few hundred years was an underlying focus on searching for ignorance in the western scientific community. The quantification of uncertainty in Bayesian inference enables clear delineation of what is unknown and what is in need of further constraint, arguably consistent with a search for ignorance. 
An example of quantifying this search for ignorance are maps of say $90 \%$ uncertainty ranges (or ranges from a NROY set in the context of history matching) in combination with space-time correlation maps. These combined maps represent a "treasure map" of where new data is most needed to better constrain the system.

This treasure map concept can be further expanded by extracting sensitivity kernels that display the dependence of some potentially measurable or proxy-inferable quantity on some system characteristic of interest. Meltwater pulse fingerprints (e.g., Mitrovica et al., 2011) and emergent constraints (e.g., Hall et al., 2019) are existing examples of this.

\subsection{A needed community test of past inference: a blind, noisy inference intercomparison}

As we move towards more rigorous inference and uncertainty assessment, there is a growing need for an independent test of methodologies. Noisy twin tests are a standard test for data-model inference algorithms. They involve adding noise to the output of a (high-complexity) "truth" simulator which then acts as input into the inferential algorithm. However, when done in-house, it is difficult for the researcher to not carry over relevant prior information from the truth model. Furthermore, if the same simulator is used for both (which has generally been the case), then even with added noise, the test is unlikely to adequately address structural discrepancy.

A complete test would therefore involve the following. Firstly, the selection of an advanced, higher-order ice sheet model, coupled climate representation, and coupled 3D glacio-isostatic adjustment, with all components at a resolution and complexity above that currently employed for ensemble-based inference of past glacial cycle ice sheet evolution. The model would be run over the last glacial cycle, preferably in a configuration that is approximately consistent with model paleo proxies given relevant uncertainties. It would be important to have the configuration replicate inferred ice and climate system variance. Secondly, participants would only have access to the model output with subtracted structural bias and added observational noise in a form that reflects available proxy data. Thirdly, the selection of a representative ice sheet that minimizes the computational burden. The Eurasian and Greenland ice sheets are the two smallest paleo ice sheets with extensive marine and terrestrial components and therefore either one could be appropriate. The Greenland ice sheet would add the significant challenge of testing appropriate ice sheet initialization and spin-up. Organizers would then compare inferential submissions against the actual chronology of the truth simulator less its structural discrepancy. Ideally, submissions would include inferences from multiple approaches and multiple error models using the same simulator to isolate the evaluation of the inferential approach and error model assessment.

The machine learning community has long relied on community-wide machine learning competitions and this is arguably one reason why machine learning has achieved such large progress over the last two decades. This blind test would entail a major community effort, especially given what is involved in complete model calibration. A major benefit is that this could thereby be used to test and refine inferential strategies, algorithms and structural discrepancy models. The results would also inform the general community of the maximum extent to which inferences to date of past ice sheet evolution might correspond to their actual evolution. 


\section{6 a community methodology research and development agenda}

This review is in part a challenge to the community and in part a statement of vision. Much is still needed to get to a point where inferential probability distributions or at least bounds are robust and make efficient use of available simulators and proxy data for paleo contexts. We've identified a number of key issues in the discussion above. They largely break down as follows:

- Emulator development: What relevant simulator outputs and internal discrepancies can be appropriately emulated, especially given computational costs of earth system simulators? To what extent can this apply to joint emulators for slow/fast versions of a simulator as well as structurally different simulators? For the latter case, how can emulators for different simulators be usefully combined? How do different emulators compare (e.g., Gaussian process versus Bayesian artificial neural networks)? Could hybrid neural network and Gaussian process emulators be formulated that encapsulate the strengths of each? Can near turn-key toolkits be developed for easy application to different simulators? To what extent can emulators for climate models be used to drive paleo ice-sheet models?

- Specification of observational uncertainties: Examples of observational uncertainties that are not yet well specified include those due to: inheritance in cosmogenic dating samples, time required for plant migration into post-glacial terrain for ${ }^{14} \mathrm{C}$ ka ages, changing tidal ranges in the context of relative sea level proxies, identification of non-analogue assemblages of foraminifera, and all the uncertainties that go into relating oxygen and deuterium isotope records to past climate. As none of the above uncertainties are likely to be well represented by a Gaussian distribution, the efficient representation of the uncertainties for likelihood application also needs consideration.

- Specification of external discrepancy: Well-formulated expert elicitation (e.g., Sexton et al., 2019) of external discrepancy is needed to both inform its specification as well as help guide simulator development to most efficiently reduce structural uncertainty.

- Specification of internal discrepancy: This includes the identification and extraction of the largest sources of internal discrepancy for individual and classes of simulators as well as the examination of the extent to which internal discrepancy varies between structurally-different simulators. There is a need to determine plausible noise structures to use for internal discrepancy experiments. Simulators also need to be configured to facilitate such noise injection.

- Parameter space sampling: The core challenge is sampling the apriori unknown and likely very small high posterior probability (or larger NROY) subspace of a large dimensional parameter space, the so-called "curse of dimensionality". This includes comparative testing of available sampling schemes for both history matching and full Bayesian inferential contexts. Such testing may inform the development of new schemes that are more efficient and/or reliable especially for exploiting parameter space reduction via restriction to active variables (c.f. 4.6).

- Forecast/retrocast: What are the pros/cons of using ensemble predictions, emulator predictions, and full Bayes? How can the design for the final history matching wave improve retrocast accuracy? How might forecast context affect stopping criteria for history matching waves? 
- Useful and tractable steps after history matching: Given the challenges of a completely rigorous and robust Bayesian inference, the development and testing of suitable approximations might permit meaningful steps beyond history matching.

- Synthesis of results from different simulators and/or different simulator analyses: Suggestions towards a systematic approach for this are presented in (Goldstein and Rougier, 2010).

Each of the above should be judged as to how they contribute towards the efficient use of the available set of simulators and computational resources to make meaningful inferences about past earth system evolution. Past experience with community simulators and model intercomparison projects indicate the progress that can be achieved from community collaboration. The challenge of working towards meaningful inference about past earth system evolution is large enough to also strongly benefit from, if not require, similar or larger scales of collaboration.

\section{Conclusions}

This review started from the premise that a defining feature of any aspect of science which is concerned about making statements about the real world is the rigorous quantification of uncertainties. Within the context of computational models, this claim can be easily supported if one recognizes that uncertainty assessment is simply the principled assessment of the relation of model results to the physical system. Without robust uncertainty estimation or, at the very least, a more limited mix of quantitative and qualitative assessment by the modeller, the reader has no basis to interpret the relevance of modelling results to the actual physical system. As our toy model illustration demonstrated above (c.f. Sect. 2.5), ignorance of structural uncertainties will generally result in model predictions that do not intersect the physical system within computed prediction limits, even if Bayes Rule is used for the inference.

The challenges in robust uncertainty assessment for complex systems, such as the earth and climate system, imply that the paleo community needs to move away from relying on a single or a few "best fit" simulations. Instead, the development and analysis of a distribution of reconstructions that bound reality within scientifically meaningful uncertainties would offer a considerable step forward. Though still requiring detailed uncertainty assessment, the focus on bounding permits one to safely err on the side of excessive uncertainty. The history matching framework described herein offers a tractable route to such inference.

This review is intended to encourage uncertainty assessment that is scientifically defensible within the paleo community and to sketch a picture of what it can look like. As should be clear, this is a highly non-trivial process. Many if not most modellers are more interested in improving model process representation, understanding model sensitivities, and analyzing modelling results than working through the numerous statistical issues and algorithms required for such meaningful uncertainty assessment. 1415 Collaboration with uncertainty quantification practitioners provides an efficient way forward. To be fruitful, such collaboration has to be from the ground up, and not just seen as an add-on near the end of a modelling project. 
https://doi.org/10.5194/cp-2021-145

Preprint. Discussion started: 17 November 2021

(c) Author(s) 2021. CC BY 4.0 License.

(c) (1)

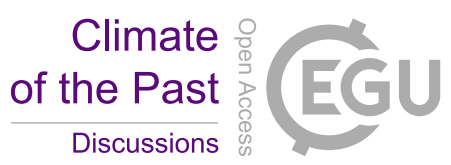

As discussed above, a key part of learning is the clear delineation of where theories, hypotheses, and models break down. Clear uncertainty assessment enables meaningful interpretation of inferences as well as the clear delineation of misfits between models and paleo data. It is these misfits that can guide both model improvement and the prioritization of future data collection. Arguably, there should therefore be as much published attention to simulator/data misfit as there is to simulator/data fits and predictions.

To conclude, for all modellers (in the general sense of modelling) making real world inferences about Earth system evolution, we reiterate some of Rougier (2007) guiding questions: What do your probabilities or inferred bounds represent? Why should a scientist believe your inferences about real world properties from simulation results or proxies more than that of someone else's? Relatedly, what are your observational and structural error models? How have they been justified? If these are not addressed, then a core question underlying many model-based studies has no answer: What does your modelling work actually contribute to our collective understanding about the physical world?

Author contributions. LT did the writing but both authors contributed equally to intellectual content.

Competing interests. The authors have no competing interests.

Acknowledgements. We gratefully acknowledge the Durham Institute for Advanced Study that provided a fellowship to LT in 2012 and thereby enabled the start of a long discussion leading to this survey. The comprehensibility of this survey strongly benefitted from comments and suggestions from Fabrice Lambert and LT's research group, including: Matt Drew, Marilena Geng, Kevin Hank, Benoit Lecavalier, Ryan Love, and especially Heather Andres. 


\section{References}

Albrecht, T., Winkelmann, R., and Levermann, A.: Glacial-cycle simulations of the Antarctic Ice Sheet with the Parallel Ice Sheet Model (PISM) Part 2: Parameter ensemble analysis, The Cryosphere, 14, 633 656, https://doi.org/10.5194/tc-14-633-2020, 2020.

Andrianakis, I., Vernon, I. R., McCreesh, N., McKinley, T. J., Oakley, J. E., Nsubuga, R. N., Goldstein, M., and White, R. G.: Bayesian History Matching of Complex Infectious Disease Models Using Emulation: A Tutorial and a Case Study on HIV in Uganda, PLoS Computational Biology, 11, e1003 968, https://doi.org/10.1371/journal.pcbi.1003968, 2015.

Andrianakis, I., McCreesh, N., Vernon, I., McKinley, T. J., Oakley, J. E., Nsubuga, R. N., Goldstein, M., and White, R. G.: Efficient History Matching of a High Dimensional Individual-Based HIV Transmission Model, SIAM/ASA Journal on Uncertainty Quantification, 5, 694 719, https://doi.org/10.1137/16m1093008, 2017.

Andrieu, C., De Freitas, N., Doucet, A., and Jordan, M.: An Introduction to MCMC for Machine Learning, in: Machine Learning, pp. 5-43, Kluwer Academic, 2003.

Annan, J. and Hargreaves, J.: Cimate prediction with imperfect models, Q.J.R. Meteorl. Soc., 128, 1-11, 2002.

Arthern, R. J.: Exploring the use of transformation group priors and the method of maximum relative entropy for Bayesian glaciological inversions, Journal of Glaciology, 61, 947 962, https://doi.org/10.3189/2015jog15j050, 2015.

Bastos, L. S. and OHagan, A.: Diagnostics for Gaussian Process Emulators, Technometrics, 51, 425438, https://doi.org/10.1198/tech.2009.08019, 2009.

Batchelor, C. L., Margold, M., Krapp, M., Murton, D. K., Dalton, A. S., Gibbard, P. L., Stokes, C. R., Murton, J. B., and Manica, A.: The configuration of Northern Hemisphere ice sheets through the Quaternary, Nature Communications, 10, https://doi.org/10.1038/s41467-019-11601-2, 2019.

Briggs, R. and Tarasov, L.: How to evaluate model derived deglaciation chronologies: A case study using Antarctica, Quat. Sci. Rev., 63, 109-127, 2013.

Briggs, R., Pollard, D., and Tarasov, L.: A data-constrained large ensemble analysis of Antarctic evolution since the Eemian, Quat. Sci. Rev., 103, 91-115, https://doi.org/10.1016/j.quascirev.2014.09.003, 2014.

Brynjarsdottir, J. and OHagan, A.: Learning about physical parameters: the importance of model discrepancy, Inverse Problems, 30,114 007, https://doi.org/10.1088/0266-5611/30/11/114007, 2014.

Chandler, R. E.: Exploiting strength, discounting weakness: combining information from multiple climate simulators, Philosophical Transactions of the Royal Society A: Mathematical, Physical and Engineering Sciences, 371, 20120 388, https://doi.org/10.1098/rsta.2012.0388, 2013.

Chang, W., Haran, M., Applegate, P., and Pollard, D.: Improving ice sheet model calibration using paleoclimate and modern data, The Annals of Applied Statistics, 10, 22742 302, https://doi.org/10.1214/16-aoas979, 2016.

Chebyshev, P.: Des valeurs moyennes, Journal de Mathématiques Pures et Appliquées, 12, 177-184, 1867.

Cornford, S., Martin, D., Graves, D., Ranken, D., Brocq, A. L., Gladstone, R., Payne, A., Ng, E., , and Lipscomb, W. H.: Adaptive mesh, finite volume modeling of marine ice sheets, J. Comput. Phys., 232, 529-549, https://doi.org/10.1016/j.bbr.2011.03.031, 2013.

Craig, P. S., Goldstein, M., Seheult, A. H., and Smith, J. A.: Pressure matching for hydrocarbon reservoirs: a case study in the use of Bayes linear strategies for large computer experiments, 1995.

Craig, P. S., Goldstein, M., Rougier, J., and Seheult, A. H.: Bayesian Forecasting for Complex Systems Using Computer Simulators, J. Amer. 
https://doi.org/10.5194/cp-2021-145

Preprint. Discussion started: 17 November 2021

(c) Author(s) 2021. CC BY 4.0 License.

(c) (i)

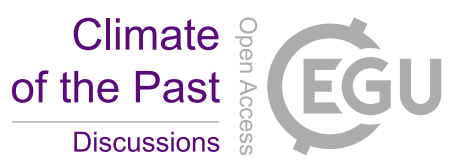

Cumming, J. and Wooff, D.: Dimension reduction via principal variables, Computational Statistics \& Data Analysis, 52, 550565, https://doi.org/10.1016/j.csda.2007.02.012, 2007.

Cumming, J. A. and Goldstein, M.: Small Sample Bayesian Designs for Complex High-Dimensional Models Based on Information Gained Using Fast Approximations, Technometrics, 51, 377 388, https://doi.org/10.1198/tech.2009.08015, 2009.

Deque, M. and Somot, S.: Weighted frequency distributions express modelling uncertainties in the ENSEMBLES regional climate experiments, Climate Research, 44, 195 209, https://doi.org/10.3354/cr00866, 2010.

Dusterhus, A., Rovere, A., Carlson, A. E., Barlow, N. L. M., Bradwell, T., Dutton, A., Gehrels, R., Hibbert, F. D., Hijma, M. P., Horton, B. P., Klemann, V., Kopp, R. E., Sivan, D., Tarasov, L., , and Tornqvist, T. E.: Palaeo sea-level and ice-sheet databases: problems, strategies, and perspectives., Clim. of the Past Discuss., 11, 2389-2404, 2015.

Eyring, V., Cox, P. M., Flato, G. M., Gleckler, P. J., Abramowitz, G., Caldwell, P., Collins, W. D., Gier, B. K., Hall, A. D., Hoffman, F. M., and et al.: Taking climate model evaluation to the next level, Nature Climate Change, 9, 102 110, https://doi.org/10.1038/s41558-018-0355-y, 2019.

Ferreira, C. J., Vernon, I., Caiado, C., Formentin, H. N., Avansi, G. D., Goldstein, M., and Schiozer, D. J.: Efficient Selection of Reservoir Model Outputs within an Emulation-Based Bayesian History-Matching Uncertainty Analysis, SPE Journal, https://doi.org/10.2118/201209-pa, 2020.

Frigg, R., Smith, L. A., and Stainforth, D. A.: An assessment of the foundational assumptions in high-resolution climate projections: the case of UKCP09, Synthese, 192, 3979-4008, https://doi.org/10.1007/s11229-015-0739-8, 2015a.

Frigg, R., Thompson, E., and Werndl, C.: Philosophy of Climate Science Part II: Modelling Climate Change, Philosophy Compass, 10, 965 977, https://doi.org/10.1111/phc3.12297, 2015b.

Gasson, E., DeConto, R., and Pollard, D.: Antarctic bedrock topography uncertainty and ice sheet stability, Geophysical Research Letters, 42, 53725 377, https://doi.org/10.1002/2015g1064322, 2015.

Gelman, A., Carlin, John, B., Stern, H. S., and Dunson, D. B. e. a.: Bayesian Data Analysis, CRC Press, 2013.

Ghahramani, Z.: Probabilistic machine learning and artificial intelligence, Nature, 521, 452 459, https://doi.org/10.1038/nature14541, 2015.

Goldstein, M.: Subjective Bayesian Analysis: Principles and Practice, Bayesian Analysis, 1, 403 420, https://doi.org/10.1214/06-ba116, 2006.

Goldstein, M. and Huntley, N.: Bayes Linear Emulation, History Matching, and Forecasting for Complex Computer Simulators, in: Handbook of Uncertainty Quantification, edited by Ghanem, R. and Higdon, D. and Owhadi, H., pp. 10-32, Springer Int., https://doi.org/10.1007/978-3-319-11259-6_14-1, 2017.

Goldstein, M. and Rougier, J.: Reified Bayesian modelling and inference for physical systems, J. Stat. Plan. Infer., $139,2010$.

Goldstein, M., Seheult, A., and Vernon, I.: Assessing Model Adequacy, in: Environmental Modelling: Finding Simplicity in Complexity, edited by J. Wainwright and M. Mulligan, pp. 435-449, John Wiley \& Sons, 2013.

Golledge, N. R., Fogwill, C. J., Mackintosh, A. N., and Buckley, K. M.: Dynamics of the last glacial maximum Antarctic ice-sheet and its response to ocean forcing, Proceedings of the National Academy of Sciences, 109, 16 052-16 056, https://doi.org/10.1073/pnas.1205385109, 2012.

Golledge, N. R., Menviel, L., Carter, L., Fogwill, C. J., England, M. H., Cortese, G., and Levy, R. H.: Antarctic contribution to meltwater pulse 1A from reduced Southern Ocean overturning, Nature Communications, 5, https://doi.org/10.1038/ncomms6107, 2014.

Goosse, H., Brovkin, V., Fichefet, T., Haarsma, R., Huybrechts, P., Jongma, J., Mouchet, A., Selten, F., Barriat, P.-Y., Campin, J.-M., Deleersnijder, E., Driesschaert, E., Goelzer, H., Janssens, I., Loutre, M.-F., Morales Maqueda, M. A., Opsteegh, T., Mathieu, P.-P., Munhoven, G., Pettersson, E. J., Renssen, H., Roche, D. M., Schaeffer, M., Tartinville, B., Timmermann, A., and Weber, S. L.: De- 
scription of the Earth system model of intermediate complexity LOVECLIM version 1.2, Geoscientific Model Development, 3, 603-633, https://doi.org/10.5194/gmd-3-603-2010, 2010.

Grant, K. M., Rohling, E. J., Bar-Matthews, M., Ayalon, A., Medina-Elizalde, M., Ramsey, C. B., Satow, C., and Roberts, A. P.: Rapid coupling between ice volume and polar temperature over the past 150,000 years, Nature, 491, 744-747, https://doi.org/10.1038/nature11593, 2012.

Hall, A., Cox, P., Huntingford, C., and Klein, S.: Progressing emergent constraints on future climate change, Nature Climate Change, 9 , 269-278, https://doi.org/10.1038/s41558-019-0436-6, 2019.

Harari, Y. N.: Sapiens, Signal, 2015.

Hebeler, F., Purves, R. S., and Jamieson, S. S. R.: The impact of parametric uncertainty and topographic error in ice-sheet modelling, J. Glaciol., 54, 899-919, 2008.

Hoare, C.: Quicksort, Commun. of the ACM, 4, 1961.

Kennedy, M. C. and O'Hagan, A.: Bayesian calibration of computer models, Journal of the Royal Statistical Society: Series B (Statistical Methodology), 63, 425-464, https://doi.org/https://doi.org/10.1111/1467-9868.00294, 2001.

Kolmogorov, A.: Foundations of Probability Theory, Chelsea Publishing, New York, 1933.

Kruschke, J. K.: Posterior predictive checks can and should be Bayesian: Comment on Gelman and Shalizi, Philosophy and the practice of Bayesian statistics, British Journal of Mathematical and Statistical Psychology, 66, 4556, https://doi.org/10.1111/j.2044-8317.2012.02063.x, 2013.

LeCun, Y., Bengio, Y., and Hinton, G.: Deep learning, Nature, 521, 436 444, https://doi.org/10.1038/nature14539, 2015.

McNeall, D. J., Challenor, P. G., Gattiker, J. R., and Stone, E. J.: The potential of an observational data set for calibration of a computationally expensive computer model, Geoscientific Model Development, 6, 17151 728, https://doi.org/10.5194/gmd-6-1715-2013, 2013.

Mitrovica, J. X., Gomez, N., Morrow, E., Hay, C., Latychev, K., and Tamisiea, M. E.: On the robustness of predictions of sea level fingerprints, Geophysical Journal International, 187, 729 742, https://doi.org/10.1111/j.1365-246x.2011.05090.x, 2011.

Neal, R. M.: Bayesian Learning for Neural Networks, vol. 118 of Lecture Notes in Statistics, Springer-Verlag, New York, 1996.

OHagan, A.: Bayesian analysis of computer code outputs: A tutorial, Reliability Engineering \& System Safety, 91, 12901300, https://doi.org/10.1016/j.ress.2005.11.025, 2006.

Pukelsheim, F.: The Three Sigma Rule, The American Statistician, 48, 88-91, https://doi.org/10.2307/2684253, 1994.

Richey, M.: The Evolution of Markov Chain Monte Carlo Methods, The American Mathematical Monthly, 117, 383-413, https://doi.org/10.4169/000298910x485923, mCMC general ref, 2010.

Rougier, J.: Probabilistic inference for future climate using an ensemble of climate model evaluations, Climate Change, 81, 247-264, 2007.

Rougier, J., Sexton, D. M. H., Murphy, J. M., and Stainforth, D.: Analyzing the Climate Sensitivity of the HadSM3 Climate Model Using Ensembles from Different but Related Experiments, Journal of Climate, 22, 35403 557, https://doi.org/10.1175/2008jcli2533.1, 2009.

Rougier, J., Goldstein, M., and House, L.: Second-Order Exchangeability Analysis for Multimodel Ensembles, Journal of the American Statistical Association, 108, 852 863, https://doi.org/10.1080/01621459.2013.802963, 2013.

Saltelli, A., Ratto, M., Andres, T., Campolongo, F., Cariboni, J., Gatelli, D., Saisana, M., and Tarantola, S.: Global Sensitivity Analysis: The Primer, John Wiley \& Sons, 2008.

Santner, T., Williams, B., and Notz, W.: The Design and Analysis of Computer Experiments, Springer-Verlag New York, 2003.

1545 Schoups, G. and Vrugt, J. A.: A formal likelihood function for parameter and predictive inference of hydrologic models with correlated, heteroscedastic, and non-Gaussian errors, Water Resources Research, 46, https://doi.org/10.1029/2009wr008933, 2010. 
https://doi.org/10.5194/cp-2021-145

Preprint. Discussion started: 17 November 2021

(c) Author(s) 2021. CC BY 4.0 License.

(c) (i)

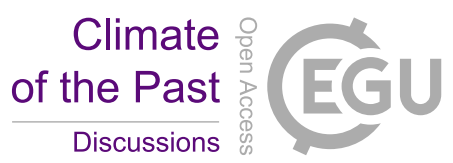

Sexton, D. M. H. and Murphy, J. M.: Multivariate probabilistic projections using imperfect climate models. Part II: robustness of methodological choices and consequences for climate sensitivity, Climate Dynamics, 38, 25432 558, https://doi.org/10.1007/s00382-011-1209-8, 2011.

Sexton, D. M. H., Karmalkar, A. V., Murphy, J. M., Williams, K. D., Boutle, I. A., Morcrette, C. J., Stirling, A. J., and Vosper, S. B.: Finding plausible and diverse variants of a climate model. Part 1: establishing the relationship between errors at weather and climate time scales, Climate Dynamics, 53, 989-1022, https://doi.org/10.1007/s00382-019-04625-3, 2019.

Sexton, D. M. H., Murphy, J. M., Collins, M., and Webb, M. J.: Multivariate probabilistic projections using imperfect climate models part I: outline of methodology, Clim. Dyn., 38, 2513-2542, https://doi.org/10.1007/s00382-011-1208-9, 2011.

Shennan, I., Long, A. J., and Horton, B. P., eds.: Handbook of Sea-level Research, AGU Wiley, 2015.

Shi, Y., Gong, W., Duan, Q., Charles, J., Xiao, C., and Wang, H.: How parameter specification of an Earth system model of intermediate complexity influences its climate simulations, Progress in Earth and Planetary Science, 6, https://doi.org/10.1186/s40645-019-0294-x, loveClim tuning paper, 2019.

Sivia, D. and Skilling, J.: Data Analysis A Bayesian Tutorial, Second Edition, Oxford University Press Inc., 2006.

Stokes, C. R., Tarasov, L., Blomdin, R., Cronin, T. M., Fisher, T. G., Gyllencreutz, R., Httestrand, C., Heyman, J., Hindmarsh, R. C., Hughes, A. L., and et al.: On the reconstruction of palaeo-ice sheets: Recent advances and future challenges, Quaternary Science Reviews, 125, 1549, https://doi.org/10.1016/j.quascirev.2015.07.016, 2015.

Tarasov, L., Dyke, A. S., Neal, R. M., and Peltier, W. R.: A data-calibrated distribution of deglacial chronologies for the North American ice complex from glaciological modeling, Earth and Plan. Sci. Let., 315-316, 30-40, 2012.

Tavassoli, Z., Carter, J., and King, P.: Errors in History Matching, SPE Journal, pp. 352-361, 2004.

Urban, N. M. and Fricker, T. E.: A comparison of Latin hypercube and grid ensemble designs for the multivariate emulation of an Earth system model, Computers \& Geosciences, 36, 746 755, https://doi.org/10.1016/j.cageo.2009.11.004, 2010.

Vernon, I., Goldstein, M., and Bower, R. G.: Galaxy formation: a Bayesian uncertainty analysis, Bayesian Analysis, 5, 619669, https://doi.org/10.1214/10-ba524, 2010.

Vernon, I., Liu, J., Goldstein, M., Rowe, J., Topping, J., and Lindsey, K.: Bayesian uncertainty analysis for complex systems biology models: emulation, global parameter searches and evaluation of gene functions, BMC Systems Biology, 12, https://doi.org/10.1186/s12918-017-0484-3, 2018.

Vysochanskii D. F., P. Y. I.: Justification of the 3 sigma rule for unimodal distributions, Theory Prob. Math. Stat., 21, 25-36, 1980.

Weigel, A. P., Knutti, R., Liniger, M. A., and Appenzeller, C.: Risks of Model Weighting in Multimodel Climate Projections, Journal of Climate, 23, 41754 191, https://doi.org/10.1175/2010jcli3594.1, 2010.

Whitehouse, P. and Tarasov, L.: Modeling and data perspectives on reconstructing Late Pleistocene ice sheets, Pages Magazine, $22,2014$.

Whitehouse, P. L., Bentley, M. J., and Brocq, A. M. L.: A deglacial model for Antarctica: geological constraints and glaciological modelling as a basis for a new model of Antarctic glacial isostatic adjustment, Quat. Sci. Rev., 32, 1-24, https://doi.org/10.1016/j.quascirev.2011.11.016, 2012.

Williamson, D., Goldstein, M., and Blaker, A.: Fast linked analyses for scenario-based hierarchies, Journal of the Royal Statistical Society: Series C (Applied Statistics), 61, 665-691, https://doi.org/10.1111/j.1467-9876.2012.01042.x, 2012.

Williamson, D., Goldstein, M., Allison, L., Blaker, A., Challenor, P., Jackson, L., and Yamazaki, K.: History matching for exploring and reducing climate model parameter space using observations and a large perturbed physics ensemble, Climate Dynamics, 41, 17031 729, https://doi.org/10.1007/s00382-013-1896-4, 2013. 
https://doi.org/10.5194/cp-2021-145

Preprint. Discussion started: 17 November 2021

(c) Author(s) 2021. CC BY 4.0 License.

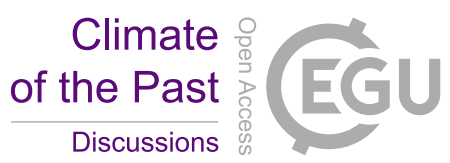

1585 Williamson, D. B., Blaker, A. T., and Sinha, B.: Tuning without over-tuning: parametric uncertainty quantification for the NEMO ocean model, Geoscientific Model Development, 10, 17891 816, https://doi.org/10.5194/gmd-10-1789-2017, 2017.

Yang, J., Reichert, P., Abbaspour, K. C., and Yang, H.: Hydrological modelling of the Chaohe Basin in China: Statistical model formulation and Bayesian inference, Journal of Hydrology, 340, 167 182, https://doi.org/10.1016/j.jhydrol.2007.04.006, 2007. 